\title{
FOURIER ANALYSIS ON THE SPHERE
}

\author{
BY
}

\author{
THOMAS O. SHERMAN
}

\begin{abstract}
A new approach to harmonic analysis on the unit sphere in $\mathrm{R}^{d+1}$ is given, closer in form to Fourier analysis on $\mathrm{R}^{d}$ than the usual development in orthonormal polynomials. Singular integrals occur in the transform formulae. The results generalize to symmetric space.
\end{abstract}

1. Introduction. Fix a positive integer $d$ and let $S$ denote the unit sphere in $\mathbf{R}^{d+1}$. Fix a point $a$ on $S$ and let $B=\{s \in S \mid s \cdot a=0\}$. ( $a$ is the "north pole" and $B$ is the "equator.") For an integer $n \geqslant 0$ and $b \in B$ define a function $e(b, n)$ on $S$ by

$$
e(b, n)(s)=(a \cdot s+i b \cdot s)^{n} \quad(s \in S) .
$$

The message of this paper is that the theory of harmonic analysis on $S$ may be formulated in a manner that so closely parallels classical Fourier analysis on abelian Lie groups as to justify our title. In this formulation the functions $e(b, n)$ and a "dual" set of functions $e_{*}(b, n)$ play the role that characters and their conjugates do on an abelian group. These functions are parameterized by $B \times N$ (where $N$ denotes the set of nonnegative integers). $B \times N$ is our Fourier transform dual space, analogous to the character group.

For suitably restricted functions $f$ on $S$ we will have formulae:

$$
\begin{gathered}
F f(b, n)=\int_{S} e(b, n)(s) f(s) d s, \\
f(s)=\int_{B \times N} e_{*}(b, n)(s) F f(b, n) d(b, n), \\
F_{*} f(b, n)=\int_{S} e_{*}(b, n)(s) f(s) d s, \\
f(s)=\int_{B \times N} e(b, n)(s) F_{*} f(b, n) d(b, n),
\end{gathered}
$$

Received by the editors May 17, 1974.

AMS (MOS) subject classifications (1970). Primary 43A85, 42A56, 42A68, 42A76, 43A75; Secondary 44A25.

Key words and phrases. Harmonic analysis, Fourier transform, sphere, symmetric space, ultraspherical polynomials, singular integral. 


$$
\int_{S} f_{1}(s) f_{2}(s) d s=\int_{B \times N} F f_{1}(b, n) F_{*} f_{2}(b, n) d(b, n) .
$$

What separates this paper from the rest of the abundant literature on this subject is the peculiar nature of the functions $e_{*}(b, n)$ which are singular with a singularity on the equator like that of $z^{-n-d+1}(z \in C)$.

$$
e_{*}(b, n)(s)=(\operatorname{sgn}(s \cdot a))^{d-1}(e(b, n+d-1)(s))^{-1} \quad(s \in S-B) .
$$

(Thus the integral in (1.3) is improper and special care is needed in its definition. Also (1.2) should be modified to say $s \notin B$.)

To continue the comparision with Fourier theory and the contrast with classical spherical theory:

Appendix 1 of this paper is devoted to the strong analogy between $e(b, n)$ on $S$ and characters of an abelian group. That appendix can be read independently of the rest of the paper and the reader is invited to glance at it at this point.

The ideas developed here are part of a general theory of Fourier analysis on Riemannian symmetric space which includes Fourier theory in the special case that the symmetric space is an abelian group.

Another special case is that of symmetric space of noncompact type. Fourier theory here was given by Helgason (see for example [2, Chapters I, II, §2]) after Harish-Chandra developed the necessary theory of zonal spherical functions. However up to now there has not appeared for the compact case a full analogue of Helgason's transform. One obstruction to a successful compact theory is the singularity of the functions which are here denoted $e_{*}(b, n)$. Thus this paper is a laboratory for the study of this singularity. (We now quickly add that our intention has been to write for a general mathematical readership and there will be no further references to symmetric space.)

Contrasts with the classical theory are evident. While $e(b, n)$ 's have played a role in the classical theory (see e.g. [3]) the goal has always been to construct and compute using an orthonormal basis of $L^{2}(S)$. The $e(b, n)$ 's span but they are not neatly orthonormal. In the present paper we abandon the emphasis on orthonormality in favor of the symmetry of (1.1)-(1.5).

To go into more detail recall the following outline of harmonic analysis on $S$ : Normalize the integral on $S$ so that $\int_{S} 1=1$. Write $H$ for $L^{2}(S) . H$ is the Hilbert space direct sum of finite dimensional subspaces $H_{n}$, each invariant and irreducible under the action of the rotation group. $H_{n}$ may be described as the space of homogeneous $n$th degree harmonic polynomials on $\mathbf{R}^{d+1}$ restricted to $S$. In $H_{n}$ there is a distinguished function $s \rightarrow P_{n}(s \cdot a)$ where $P_{n}$ is a polynomial of degree $n$ on $\mathbf{R}$, with $P_{n}(1)=1$. The orthogonal projection $H \rightarrow H_{n}$ is given by $f \rightarrow f_{n}$ where 


$$
f_{n}(s)=\operatorname{dim}\left(H_{n}\right) \int_{S} f\left(s_{1}\right) P_{n}\left(s_{1} \cdot s\right) d s_{1} .
$$

Our contribution is to analyse (1.6) into two stages: Stage I given by (1.1) (or (1.3)) and Stage II given by (1.7) (or (1.8)) below.

$$
\begin{aligned}
& f_{n}(s)=\operatorname{dim}\left(H_{n}\right) \int_{B} e_{*}(b, n)(s) F f(b, n) d b, \\
& f_{n}(s)=\operatorname{dim}\left(H_{n}\right) \int_{B} e(b, n)(s) F_{*} f(b, n) d b .
\end{aligned}
$$

(1.2) is a corollary of (1.7) and (1.4) is a corollary of (1.8) once it is explained that the measure on $B \times N$ is the product of the normalized rotation invariant measure on $B$ with the measure on $N$ which assigns to the singleton $\{n\}$ the value $\operatorname{dim}\left(H_{n}\right) .(1.7)$ and (1.8) follow from (1.6) and the following result:

$$
P_{n}\left(s \cdot s_{1}\right)=\int_{B} e_{*}(b, n)(s) e(b, n)\left(s_{1}\right) d b
$$

where $s \in S-B$ and $s_{1} \in S$.

(1.9) may be fairly regarded as the key to the paper.

The paper is organized as follows:

$\S 2$ is a brief survey of the classical theory without proofs. The goal is to establish notation and help isolate what is new from what is well known.

$\S 3$ is a rigorous statement of the main results without proof, namely (1.1)(1.5) and (1.7)-(1.9).

$\S 4$ proves (1.9).

$\S 5$ is devoted to the definition and theory of the improper integral in (1.3). The hope is that the theory will prove to be computationally effective. However, the section is long and should probably be skimmed lightly on first reading.

$\S 6$ combines the results of $\S \S 4$ and 5 into proofs of the statements in $\S 3$.

Numbering up to this point is as follows: $(x . y)$ or $(x . y . z)$ refers to a numbered formula or equation in $\S x$. The format $(x . y)$ is used with $x=1,2$. (x.y.z) is used with $x=3$ through 6 . In this range $x . y$ (without parenthesis) refers to the $y$ th numbered result (i.e., definition, lemma, or theorem) in $\S x$ and (x.y.z) is the $z$ th formula within that result.

Appendix 1 examines the analogy between $e(b, n)$ on $S$ and characters on abelian Lie groups.

Appendix 2 is a miscellany of results leaning toward a calculus of $F$ and $F_{*}$.

2. Notation and classical results. Fix a positive integer $d$. $S$ denotes the unit sphere in $\mathbf{R}^{d+1}$. There is a unique rotation-invariant Borel measure on $S$ such that the measure of $S$ is 1 . This measure on $S$ is always understood (with one brief exception in Lemma 5.2 that will be clearly marked): $\int_{S} f$ or $\int_{S} f(s) d s$ 
denotes integration with respect to this measure.

Let $H=L^{2}(S) . H$ is the Hilbert space direct sum of finite dimensional subspaces $H_{n}, n$ an integer $\geqslant 0$. Let $H_{n}$ denote the space of harmonic homogeneous polynomials of degree $n$ on $\mathbf{R}^{d+1}$. $H_{n}$ is obtained by restricting the functions in $H_{n}$ to $S$. The restriction map from $H_{n}$ to $H_{n}$ is a bijection. $\operatorname{dim}\left(H_{n}\right)$ has the generating function

$$
\sum_{0}^{\infty} \operatorname{dim}\left(H_{n}\right) t^{n}=(1+t)(1-t)^{-d}
$$

thus

$$
\begin{aligned}
\operatorname{dim}\left(H_{n}\right) & =(n+d-1) ! / n !(d-1) !+(n+d-2) ! /(n-1) !(d-1) ! \\
& =(n+d) ! / n ! d !-(n+d-2) ! /(n-2) ! d ! .
\end{aligned}
$$

There is a unique function $P_{n}=P_{n, d}$ on $[-1,1]$ with the following two properties:

(2.1) $P_{n}(1)=1$;

(2.2) $s \rightarrow P_{n}\left(s \cdot s_{1}\right)$ is in $H_{n}\left(s_{1}\right.$ fixed, $\left.s \in S\right) . P_{n}$ is in fact a polynomial of degree $n$ and except for a constant factor equals the Gegenbauer polynomial $C_{n}^{\lambda}$ where $\lambda=(d-1) / 2$ or the Jacobi polynomial $P_{n}^{(\alpha, \alpha)}$ where $\alpha=(d-2) / 2$. It is given by

$$
P_{n}=Q_{n} / Q_{n}(1)
$$

where

$$
\begin{aligned}
& Q_{n}(t)=\left(1-t^{2}\right)^{-\alpha}(d / d t)^{n}\left(1-t^{2}\right)^{n+\alpha}, \quad \alpha=(d-2) / 2, \\
& Q_{n}(1)=(-2)^{n} \Gamma(n+\alpha+1) / \Gamma(\alpha+1) .
\end{aligned}
$$

The orthogonal projection of $f \in H$ to $f_{n} \in H_{n}$ is given by

$$
f_{n}\left(s_{1}\right)=\operatorname{dim}\left(H_{n}\right) \int_{S} f(s) P_{n}\left(s_{1} \cdot s\right) d s .
$$

Let $G$ denote the group of rotations of $\mathbf{R}^{d+1}$ around the origin. $g \in G$ acts on a function $f$ (on $S$ or $\mathbf{R}^{d+1}$ ) by sending $f$ to $f^{g}$ where $f^{g}(x)=f(g x)$. Note that $f^{g \gamma}=\left(f^{g}\right)^{\gamma}(g, \gamma \in G)$. Thus $G$ has a representation on $H$ which is unitary.

The spaces $H_{n}$ may be characterized as the irreducible components of $H$ under the above representation of $G$, taken in increasing order of $\operatorname{dim}\left(H_{n}\right)$.

Let $D$ denote $C^{\infty}(S)$ equipped with the topology of uniform convergence on $S$ in all derivatives. Let $D^{\prime}$ denote the space of continuous linear functionals on $D$. We write elements of $D^{\prime}$ as though they were functions integrated against elements of $D$. Thus if $f^{\prime} \in D^{\prime}$ and $f \in D$ then the value of $f^{\prime}$ at $f$ is denoted 
$\int_{S} f^{\prime} f=\int_{S} f f^{\prime} . H$ has a natural embedding in $D^{\prime}$ which is consistent with this notation. Thus $H_{n} \subset D \subset H \subset D^{\prime}$.

For $f^{\prime} \in D^{\prime}$ we may define $f_{n}^{\prime} \in H_{n}$ by formula (2.3). Then we have $\int_{S} f_{n}^{\prime} f=\int_{S} f_{n}^{\prime} f_{n}=\int_{S} f^{\prime} f_{n}\left(f^{\prime} \in D^{\prime}, f \in D\right)$.

THEOREM. Let $f \in D$. Then the series $\Sigma_{0}^{\infty} f_{n}$ converges to $f$ in the topology of $D$.

As a general reference for this section see [3].

3. Statement of results. Fix a point $a$ in $S$, once and forever. Let $B=$ $\{s \in S \mid s \cdot a=0\} . B$ is the unit sphere in $a^{\perp}$. The rotation invariant integral on $B$ with $\int_{B} 1=1$ is denoted $\int_{B} f$ or $\int_{B} f(b) d b$.

Let $N$ denote the nonnegative integers. Any sum unless otherwise indicated is over $n \in N$.

For $(b, n) \in B \times N$ we have two functions on $S$ and $S-B$ respectively, defined as follows.

$$
\begin{gathered}
e(b, n)(s)=(a \cdot s+i b \cdot s)^{n} \quad(s \in S), \\
e_{*}(b, n)(s)=(\operatorname{sgn}(s \cdot a))^{d-1}(e(b, n+d-1)(s))^{-1} \quad(s \in S-B) .
\end{gathered}
$$

Definition 3.1. For any $f^{\prime} \in D^{\prime}$ we define $F f^{\prime}(b, n)=\int_{S} f^{\prime} e(b, n)$.

DEFinition 3.2. Let $\rho$ be a $C^{\infty}$ function on $\mathbf{R}$ whose support contains 0 and such that the support of $1-\rho$ is compact. For $t \in \mathbf{R}$ let $r_{t}$ be the $C^{\infty}$ function defined on $S$ by

$$
r_{t}(s)=\rho(t(s \cdot a)), \quad s \in S .
$$

For $f^{\prime} \in D^{\prime}$ define $F_{t} f^{\prime}(b, n)$ by

$$
F_{t} f^{\prime}(b, n)=\int_{S} f^{\prime} r_{t} e_{*}(b, n) .
$$

If $\lim _{t \rightarrow \infty} F_{t} f^{\prime}(b, n)$ exists it is denoted $F_{*} f^{\prime}(b, n)$.

This definition depends on the choice of $\rho$ which will be considered fixed (but not further specified).

Remark. Both definitions apply to $f \in L^{1}(S)$ or $f \in D$ since $D \subset L^{1}(S)$ $\subset D^{\prime}$.

Lemma 3.3. If $f \in D$ then $F_{*} f(b, n)$ exists for all $(b, n) \in B \times N$. For fixed $n, F_{*} f(b, n)$ is a continuous function of $b$. In fact as $t \rightarrow \infty, F_{t} f(b, n)$ converges to $F_{*} f(b, n)$ uniformly on $B$. The map $f \rightarrow F_{*} f(b, n)$ is in $D^{\prime}$ for fixed $(b, n)$.

THEOREM 3.4. Fix $n \in N$. Then for $f^{\prime} \in D^{\prime}$ we may compute $f_{n}^{\prime} \in H_{n}$ by 


$$
f_{n}^{\prime}(s)=\operatorname{dim}\left(H_{n}\right) \int_{B} F f^{\prime}(b, n) e_{*}(b, n)(s) d b
$$

where $s \in S-B$. (Note. $F f^{\prime}(b, n)$ is a polynomial function of $b$ of degree $\leqslant n$. Also for $s \in S-B$ the function $e_{*}(b, n)(s)$ is analytic in $b$. Thus the integral is well defined.)

Corollary 3.5. Let $f \in H$. Then

$$
f(s)=\sum \operatorname{dim}\left(H_{n}\right) \int_{B} F f(b, n) e_{*}(b, n)(s) d b
$$

with the series converging in the norm of $H$. If $f \in D$ then the series converges uniformly on $S-B$.

THEOREM 3.6. Let $f \in D$. Then for all $s \in S$

$$
f_{n}(s)=\operatorname{dim}\left(H_{n}\right) \int_{B} F_{*} f(b, n) e(b, n)(s) d b .
$$

Corollary 3.7. Let $f \in D$. Then

$$
f(s)=\sum \operatorname{dim}\left(H_{n}\right) \int_{B} F_{*} f(b, n) e(b, n)(s) d b
$$

where the series converges in $D$.

THEOREM 3.8. Let $f \in D$ and $f^{\prime} \in D^{\prime}$. Then

$$
\int_{S} f f^{\prime}=\sum \operatorname{dim}\left(H_{n}\right) \int_{B} F_{*} f(b, n) F f^{\prime}(b, n) d b .
$$

The corollaries follow by combining the theorems with classical results (see §2). The theorems in turn hinge on the formula (2.3), Lemma 3.3, and the following lemma which is the most important new result.

Key Lemma 3.9. Fix $n \in N, s_{1} \in S$ and $s \in S-B$. Then

$$
P_{n}\left(s_{1} \cdot s\right)=\int_{B} e(b, n)\left(s_{1}\right) e_{*}(b, n)(s) d b .
$$

This formula generalizes to arbitrary Riemannian symmetric space. Helgason proved it by integral means for noncompact symmetric space and used it to obtain a second proof of his Fourier transform theory. That proof does not work for compact symmetric space, where the formula appears to be new even for the sphere in $\mathbf{R}^{\mathbf{3}}$.

4. The Key Lemma. This section is devoted to a proof of Lemma 3.9. We begin with the case $d=1$ which turns out to be elementary (Lemma 4.1). Thereafter we assume $d \geqslant 2$. Our next step is to establish the case of $s=a$ (Lemma 4.2). This also is easy and not original; the resulting formula is classical and a 
special case of a general type of formula given by Harish-Chandra for zonal spherical functions; in case $d=2$ it is called "Laplace's integral of the first kind."

Then begins the serious work on the Key Lemma. It will be helpful if we give here an outline of the argument with some of the notation to be used.

After Lemma 4.1 fix the integers $d \geqslant 2$ and $n \in N$. Let

$$
I\left(s_{1}, s\right)=\int_{B} e(b, n)\left(s_{1}\right) e_{*}(b, n)(s) d b \quad\left(s_{1} \in S, s \in S-B\right) .
$$

The problem is to prove that $I\left(s_{1}, s\right)=P_{n}\left(s_{1} \cdot s\right)$. By Lemma 4.2, $I\left(s_{1}, a\right)=$ $P_{n}\left(s_{1} \cdot a\right)$.

Recall that we are using $G$ to denote the group of rotations in $\mathbf{R}^{d+1}$ around the origin. Consider

$$
i(g)=i\left(g ; s_{1}, s\right)=I\left(g s_{1}, g s\right) .
$$

If $I\left(s_{1}, s\right)=P_{n}\left(s_{1} \cdot s\right)$ is true then $i$ is locally constant on $G$. Conversely if we can show that $i$ is locally constant we will be very close to proving that $I\left(s_{1}, s\right)$ is a function of $s_{1} \cdot s$ and this by Lemma 4.2 will be enough.

The heart of the proof then is the demonstration that $i$ is locally constant.

Let $\boldsymbol{g}$ denote the real Lie algebra of $G$. For $x \in \mathfrak{g}$ let $D_{x}$ denote the corresponding differential operator on $G$ acting on the left. We will show (Lemma 4.3) that $D_{x} i=0$. The idea is to take $D_{x}$ under the integral sign, shift the derivative over to the $b$-variable and show that the resulting integral of a derivative is zero.

Lemma 4.1. Lemma 3.9 holds in case $d=1$. More explicitly: $P_{n}$ is the Tchebychev polynomial of degree $n ; a=(1,0) ; B=\{(0,1),(0,-1)\} ;$ if $s_{1}=$ $\left(x_{1}, y_{1}\right), s=(x, y)$ then $e((0, \pm 1), n)\left(s_{1}\right)=\left(x_{1} \pm i y_{1}\right)^{n}$ and $e_{*}((0, \pm 1), n)(s)=$ $(x \pm i y)^{-n}$. Then our assertion is

$$
\begin{aligned}
P_{n}\left(x_{1} x+y_{1} y\right)=1 / 2( & ((0,1), n)\left(s_{1}\right) e_{*}((0,1), n)(s) \\
& \left.+e((0,-1), n)\left(s_{1}\right) e_{*}((0,-1), n)(s)\right) .
\end{aligned}
$$

Proof. Let $x_{1}+i y_{1}=\exp \left(i \theta_{1}\right)$ and $x+i y=\exp (i \theta)$. Then

$$
P_{n}\left(x_{1} x+y_{1} y\right)=P_{n}\left(\cos \left(\theta-\theta_{1}\right)\right)=\cos \left(n\left(\theta-\theta_{1}\right)\right) \text {. }
$$

On the other side we have

$$
e((0,1), n)\left(s_{1}\right)=\exp \left(\operatorname{in} \theta_{1}\right), \quad e((0,-1), n)(s)=\exp (-\operatorname{in} \theta)
$$

and so on. Thus the formula becomes

$$
\cos \left(n\left(\theta-\theta_{1}\right)\right)=1 / 2\left(\exp \left(i n \theta_{1}\right) \exp (-i n \theta)+\exp \left(-i n \theta_{1}\right) \exp (i n \theta)\right)
$$

which is of course true. 
From now on assume $d \geqslant 1$ and $n \in N$ are fixed integers.

Lemma 4.2 [3, Theorem 7]. Let $s \in S$. Then

$$
P_{n}(s \cdot a)=\int_{B} e(b, n)(s) d b .
$$

Proof. For each $b \in B, e(b, n) \in H_{n}$ since $p(x)=(x \cdot a+i x \cdot b)^{n}$ is harmonic on $\mathbf{R}^{d+1}$ and $e(b, n)=p \mid S$.

The function $b \rightarrow e(b, n)$ is continuous from $B$ to the finite dimensional vector space $H_{n}$. Thus $\int_{B} e(b, n) d b \in H_{n}$. Evaluation at $s \in S$ is a linear functional on $H_{n}$ so the function

$$
\phi(s)=\int_{B} e(b, n)(s) d b=\left(\int_{B} e(b, n) d b\right)(s)
$$

is in $H_{n}$.

Now $\phi$ is symmetric around the axis $R a$. (Indeed if $K \subset G$ is the group of rotations fixing $a$ then $\phi(k s)=\phi(s)(k \in K, s \in S)$. This follows from $e(b, n)(k s)$ $=e\left(k^{-1} b, n\right)(s)$ and the $K$-invariance of $\int_{B}$. $)$ This symmetry implies that for some function $R$ on $[-1,1], \phi(s)=R(s \cdot a)$. Also $R(1)=1$ since $e(b, n)(a)=1$ and $\int_{B} 1=1$. Thus $R$ satisfies (2.1) and (2.2) with $s_{1}=a$, proving $R=P_{n}$.

We will write $I\left(s_{1}, s\right)=\int_{B} e(b, n)\left(s_{1}\right) e_{*}(b, n)(s) d b$ when $s_{1} \in S$ and $s \in$ $S-B$. From the lemma just proved it suffices to show that $I\left(s_{1}, s\right)$ is really a function of $s_{1} \cdot s$, for then that function is necessarily $P_{n}$. To this end let $G(s)=$ $\{g \in G \mid g s \notin B\}$.

The remainder of the proof shows that $i(g)=i\left(g ; s_{1}, s\right)=I\left(g s_{1}, g s\right)$ is constant on $G(s)$. (The reason that this is enough is simply that if $\left(s_{1}^{\prime}, s^{\prime}\right)$ is another pair in $S \times(S-B)$ with $s_{1} \cdot s=s_{1}^{\prime} \cdot s^{\prime}$ then there is $g \in G\left(s_{2}\right)$ such that $g s_{1}=s_{1}^{\prime}, g s=s^{\prime}$.) Our first and hardest task is to show that $i$ is locally constant. This we do by differential means.

The real Lie algebra $g$ of $G$ will be identified with the skew-symmetric linear transforms on $\mathbf{R}^{d+1}$. $x \in \mathbb{g}$ gives rise to the differential operator $D_{x}$ on $G$ :

$$
D_{x} f(g)=-(d / d t) f(\exp (t x) g)_{\mid t=0}
$$

where $f$ is differentiable in a neighborhood of $g$ in $G$. To show that $i$ is locally constant it is enough to establish

Lemma 4.3. For all $x \in \mathfrak{g}, D_{x} i=0$ on $G(s)$.

As stated earlier our method of proof is to shift $D_{x}$ inside the integral sign to obtain

$$
D_{x} i(g)=\int_{B} D_{x}\left(e(b, n)\left(g s_{1}\right) e_{*}(b, n)(g s)\right) d b
$$


Then we shift the derivative from the variable $g$ to the variable $b$. We wish to study this shift from $g$ to $b$ in advance of the proof of 4.3 .

Recall that $B$ is the unit sphere in $a^{\perp}$. We identify $a^{\perp}$ with $\mathbf{R}^{d}$.

Definition. By a "real tangent field on $B$ " we mean a function $\phi: B \rightarrow$ $\mathbf{R}^{d}$ such that for all $b \in B, \phi(b) \cdot b=0$. By a "complex tangent field on $B$ " we mean a function $\phi_{1}+i \phi_{2}: B \rightarrow C^{d}$ where $\phi_{1}$ and $\phi_{2}$ are real tangent fields. If $\phi=\phi_{1}+i \phi_{2}$ is a complex tangent field and $f$ is a $C^{1}$ function on $B$ then $\phi$ acts as a differential operator on $f$ (notation. $\phi \# f$ ) as follows: extend $f$ to a $C^{1}$ function in an open neighborhood of $B$ in $\mathbf{R}^{d}$. Then

$$
\left(\phi_{1} \# f\right)(b)=(d / d t) f\left(b+t \phi_{1}(b)\right)_{\mid t=0} .
$$

$\phi_{2} \# f$ is similarly defined and $\phi \# f=\phi_{1} \# f+i \phi_{2} \# f$.

Now given $x \in \mathfrak{g}$ we assign a complex tangent field $\beta_{x}$ on $B$ by the formula

$$
\begin{aligned}
\beta_{x}(b) & =x(b)-(x(b) \cdot a) a-i(x(a)-(x(a) \cdot b) b) \\
& =-i(x(a)+i x(b)-((x(a)+i x(b)) a) a-((x(a)+i x(b)) \cdot b) b) .
\end{aligned}
$$

Geometrically $\beta_{x}$ is the orthogonal projection of $-i x(a+i b)$ onto the complex tangent plane to $B$ at $b$.

Lemma 4.4. Fix $s \in S$. For $g \in G, b \in B$ let $\phi(g, b)=e(b, 1)(g s)$. Let $m$ be an integer. If $m$ is negative restrict $g$ to $G(s)$. Then

$$
D_{x} \phi^{m}=-i m(x(a) \cdot b) \phi^{m}+\beta_{x} \# \phi^{m}
$$

where $D_{x}$ acts with respect to $g$ and $\beta_{x} \#$ acts with respect to $b$.

Before giving the proof, some motivation can be obtained from

Corollary 4.5. For $s_{1} \in S, b \in B, n \in N, x \in \mathfrak{g}, g \in G$,

(4.5.1) $D_{x} e(b, n)\left(g s_{1}\right)=-i n(x(a) \cdot b) e(b, n)\left(g s_{1}\right)+\beta_{x} \# e(b, n)\left(g s_{1}\right)$.

If $g \in G(s)$ then:

$(4.5 .2) D_{x} e_{*}(b, n)(g s)=i(n+d-1)(x(a) \cdot b) e_{*}(b, n)(g s)+\beta_{x} \# e_{*}(b, n)(g s)$.

$$
\begin{aligned}
D_{x}\left(e(b, n)\left(g s_{1}\right) e_{*}(b, n)(g s)\right)= & \left(i(d-1)(x(a) \cdot b)+\beta_{x} \#\right) \\
& \cdot\left(e(b, n)\left(g s_{1}\right) e_{*}(b, n)(g s)\right) .
\end{aligned}
$$

Proof of Corollary 4.5. $e(b, n)(g s)=\phi^{n}(g, b)$; and $e_{*}(b, n)(g s)$ is locally (on $G(s) \times B$ ) a constant $\left(+1\right.$ or -1 ) multiple of $\phi^{-n-d+1}(g, b)$. (4.5.1) and (4.5.2) now follow immediately from 4.4. (4.5.3) follows from (4.5.1) and (4.5.2) by Leibnitz' rule for differentiating a product.

Proof of Lemma 4.4. First consider $m=1$. 


$$
\begin{aligned}
D_{x} \phi(g, b) & =-a \cdot x(g s)-i b \cdot x(g s)=x(a) g s+i x(b) g s \\
& =-i(x(a) \cdot b)(a \cdot g s+i b \cdot g s)+i \beta_{x}(b) \cdot g s \\
& =-i(x(a) \cdot b) \phi(g, b)+\beta_{x} \# \phi(g, b)
\end{aligned}
$$

proving the case $m=1$. The case of $m$ an arbitrary integer follows from this one by

$$
\begin{aligned}
D_{x} \phi^{m} & =m \phi^{m-1} D_{x} \phi=m \phi^{m-1}\left(-i(x(a) \cdot b) \phi+\beta_{x} \# \phi\right) \\
& =-i m(x(a) \cdot b) \phi^{m}+\beta_{x} \# \phi^{m} .
\end{aligned}
$$

Lemma 4.6. Let $f \in C^{1}(B)$ and $x \in \mathrm{g}$. Then

$$
\int_{B} i(d-1)(x(a) \cdot b) f(b)+\beta_{x} \# f(b) d b=0 .
$$

Proof. This is an application of the divergence theorem once we recognize that the divergence of the vector field $\beta_{x}$ on the manifold $B$ at the point $b$ is $i(d-1) x(a) \cdot b$. To see this consider the real and imaginary parts of $\beta_{x}$ separately.

The real part of $\beta_{x}$ is $b \rightarrow x(b)-(x(b) \cdot a) a$. This map from $B$ to $\mathbf{R}^{d}$ extends uniquely to a linear map of $\mathbf{R}^{d}$ to $\mathbf{R}^{d}$ which is skew-symmetric. The flow of such a vector field on $B$ is a rotation which is volume preserving. Clearly then the divergence of the real part of $\beta_{x}$ is 0 .

The imaginary part of $\beta_{x}$ is $-i \gamma_{x}$ where $\gamma_{x}(b)=x(a)-(x(a) \cdot b) b$. We must show that $\operatorname{div}\left(\gamma_{x}\right)(b)=-(d-1) x(a) \cdot b$. Consider the following extension of $\gamma_{x}$ to a vector field (again denoted $\gamma_{x}$ ) on $\mathbf{R}^{d}: \gamma_{x}(v)=x(a)(v \cdot v)-(x(a) \cdot v) v$ $\left(v \in \mathbf{R}^{d}\right)$. Taken as a vector field on $\mathbf{R}^{d}$ the divergence of $\gamma_{x}$ is easily seen to be $-(d-1) x(a) \cdot v$. Now if we compute $\operatorname{div}\left(\gamma_{x}\right)$ on $\mathbf{R}^{d}$ in polar coordinates and evaluate at $b \in B$ we obtain the sum of a radial term (which is, however, 0 since $\gamma_{x}(v) \cdot v=0$ ) and a spherical term which is just the divergence of $\boldsymbol{\gamma}_{x}$ as a vector field on $B$. Thus on $B, \operatorname{div}_{B}\left(\gamma_{x}\right)(b)=-(d-1) x(a) \cdot b$.

Proof of Lemma 4.3. For $g \in G(s)$ let

$$
f(g, b)=e(b, n)\left(g s_{1}\right) e_{*}(b, n)(g s) .
$$

By (4.5.3) of Lemma 4.5

$$
D_{x} f(g, b)=i(d-1)(x(a) \cdot b) f(g, b)+\beta_{x} \# f(g, b) .
$$

Thus by Lemma $4.6, \int_{B} D_{x} f(g, b) d b=0$. Then

$$
D_{x} i(g)=D_{x} \int_{B} f(g, b) d b=\int_{B} D_{x} f(g, b) d b=0 .
$$

We have proved that $i$ is locally constant on $G(s)$. It remains only to prove 
that it is globally constant. To do this write $G(s)=G_{+} \cup G_{-}$where $G_{+}$is the set of all $g \in G(s)$ which carry $s$ into the component of $S-B$ containing $s ; G_{-}$is the complement: the set of all $g \in G(s)$ which carry $s$ into the opposite component of $S-B$.

Lemma 4.7. $i$ is constant on $G_{+}$and on $G_{-}$.

Proof. Let $G_{0}$ stand for either $G_{+}$or $G_{-}$consistently. The set $\left\{\left(g s_{1}\right.\right.$, $\left.g s) \mid g \in G_{0}\right\}$ is a component of $S \times(S-B)$. Let $g$ and $g^{\prime}$ be two elements of $G_{0}$. Then there is a third element $g^{\prime \prime}$ of $G_{0}$ connected to $g$ by a continuous curve in $G_{0}$ such that $\left(g^{\prime \prime} s_{1}, g^{\prime \prime} s\right)=\left(g^{\prime} s_{1}, g^{\prime} s\right)$. $i(g)=i\left(g^{\prime \prime}\right)$ because $i$ is locally constant; and

$$
i\left(g^{\prime \prime}\right)=I\left(g^{\prime \prime} s_{1}, g^{\prime \prime} s\right)=I\left(g^{\prime} s_{1}, g^{\prime} s\right)=i\left(g^{\prime}\right) \text {. }
$$

So $i(g)=i\left(g^{\prime}\right)$ proving $i$ is constant on $G_{0}$.

LEMMA 4.8. $i$ is constant on $G(s)$.

Proof. It remains only to show that $i$ takes the same value on $G_{+}$and $G_{-}$. To this end we compare $i$ at $I \in G_{+}$and $-I \in G_{-}$where $I$ denotes the identity transform in $G$.

$$
\begin{aligned}
i(-I)= & \int_{B} e(b, n)\left(-s_{1}\right) e_{*}(b, n)(-s) d b \\
= & \int_{B}(-1)^{n}\left(a \cdot s_{1}+i b \cdot s_{1}\right)^{n}(\operatorname{sgn}(-s \cdot a))^{d-1} \\
& \cdot(-1)^{-n-d+1}(s \cdot a+i s \cdot b)^{-n-d+1} d b \\
= & \int_{B} e(b, n)\left(s_{1}\right) e_{*}(b, n)(s) d b=i(I) .
\end{aligned}
$$

Proof of Key Lemma 3.9. Lemma 4.8 states that for all $g \in G$ such that $g s \notin B i(g)=I\left(g s_{1}, g s\right)$ is independent of $g$. Now $\left\{\left(g s_{1}, g s\right) \mid g \in G(s)\right\}$ is precisely the set of points $\left(s_{1}^{\prime}, s^{\prime}\right)$ in $S \times S-B$ for which $s_{1}^{\prime} \cdot s^{\prime}=s_{1} \cdot s$. Thus $I\left(s_{1}, s\right)$ really depends only on $s_{1} \cdot s$, i.e. there is a function $R$ on $[-1,1]$ such that $I\left(s_{1}, s\right)=R\left(s_{1} \cdot s\right)$. By Lemma $4.2, R\left(s_{1} \cdot a\right)=P_{n}\left(s_{1} \cdot a\right)\left(s_{1} \in S\right)$. Thus $R=$ $P_{n}$ and we have $I\left(s_{1}, s\right)=P_{n}\left(s_{1} \cdot s\right)$.

As a final remark it is interesting to note what the Key Lemma says when $s_{1}=a$. As in 4.2 we get $P_{n}(s \cdot a)=\int_{B} e_{*}(b, n)(s) d b$. In case $d=2$ this is called Laplace's integral of the second kind. For $d>2$ it appears to be new. The notable thing here is the singularity of $e_{*}$ versus the regularity of $\boldsymbol{P}_{n}$.

5. A singular integral. In this section we study the divergent improper integral $\int_{S} f e_{*}(b, n)$. With reference to Definition 3.2 we could say that $\int_{S} f e_{*}(b, n)$ 
is defined as $F_{*} f(b, n)$, provided the latter exists. Then we should give an existence theory and a computational method. In fact our existence theory lends itself directly to computation.

Let $\mathbf{D}$ denote the closed unit disk in $\mathbf{C}$. On $\mathbf{D}$ consider the function $\epsilon_{n, d}(z)$ $=(\operatorname{sgn}(\operatorname{Re}(z)))^{d-1} z^{-n-d+1}$. There is more than a formal analogy between the integral $\int_{S} f e_{*}(b, n)$ and the integral $\int_{\mathrm{D}} \phi \epsilon_{n, d}$ (Lebesgue measure is understood on D) as we will see in Corollary 5.3. Just for now consider the problem of defining $\int_{\mathrm{D}} \phi \epsilon_{n, d} \cdot \epsilon_{n, d}$ is so singular at $z=0$ that if $\phi$ is, say, continuous and $\phi(0) \neq 0$ then $\phi \epsilon_{n, d}$ is certainly not in $L^{1}$ (D). Nevertheless if $\phi$ is sufficiently smooth at $z=0$ we can assign a meaning to $\int_{D} \phi \epsilon_{n, d}$ (see Lemma 5.1). After showing how to do that we turn to the connection between this singular integral on $\mathbf{D}$ and our original problem on $S$. We give a method for reducing the problem on $S$ to the problem on D via the map $e(b, 1): S \rightarrow \mathrm{D}$.

Recall the function $\rho$ of Definition 3.2. It is defined on $R$, is of class $C^{\infty}$, vanishes in a neighborhood of 0 and is identically 1 outside a compact set. Define a function $\rho_{t}$ on $\mathbf{C}$ for $t \in \mathbf{R}$ :

$$
\rho_{t}(z)=\rho(t x) \quad(x+i y=z \in \mathbf{C}) .
$$

LEMMA 5.1. Let $\phi \in L^{1}$ (D). Suppose for some open neighborhood $U$ of 0 in Int(D) we have $\phi \mid U \in C^{n+d-2}(U)$. Then $\lim _{t \rightarrow \infty} \int_{D} \rho_{t} \phi \epsilon_{n, d}$ exists and is independent of the choice of $\rho$.

Proof. Choose a $C^{\infty}$ partition of unity for D: $1=\psi_{0}+\psi_{1}$ where $\psi_{0}$ vanishes in a neighborhood of 0 and $\operatorname{supp}\left(\psi_{1}\right) \subset U$.

Then $\psi_{0} \epsilon_{n, d}$ is smooth so $\lim _{t \rightarrow \infty} \int_{\mathrm{D}} \rho_{t} \phi \psi_{0} \epsilon_{n, d}=\int_{\mathrm{D}} \phi \psi_{0} \epsilon_{n, d}$.

Next let $Y=-i \partial / \partial y(z=x+i y$ in $\mathrm{D})$ and $\epsilon_{2-d, d}(z)=(\operatorname{sgn}(x))^{d-1} z^{-1}$.

Then $\epsilon_{n, d}=(k !)^{-1}(Y)^{k} \epsilon_{2-d, d}$ where $k=n+d-2$.

Using the fact that $\psi_{1} \phi \in C^{k}(\operatorname{Int}(D))$ with support in $\operatorname{Int(D)}$ we have

$$
\begin{aligned}
\int_{\mathrm{D}} \rho_{1} \psi_{1} \phi \epsilon_{n, d} & =\int_{\mathrm{D}}(k !)^{-1}\left((-Y)^{k} \rho_{t} \psi_{1} \phi\right) \epsilon_{2-d, d} \\
& =\int_{\mathrm{D}}(k !)^{-1} \rho_{t}\left((-Y)^{k} \psi_{1} \phi\right) \epsilon_{2-d, d} .
\end{aligned}
$$

Since $\epsilon_{2-d, d} \in L^{1}(\mathrm{D})$ and $\rho_{t} \rightarrow 1$ boundedly pointwise (except at $x=0$ ) the limit as $t \rightarrow \infty$ of the above integrals exists and equals $(k !)^{-1} \int_{D}\left((-Y)^{k} \psi_{1} \phi\right) \epsilon_{2-d, d}$. Thus

(5.1.2) $\lim _{t \rightarrow \infty} \int_{\mathrm{D}} \rho_{t} \phi \epsilon_{n, d}=\int_{\mathrm{D}} \psi_{0} \phi \epsilon_{n, d}+(k !)^{-1} \int_{\mathrm{D}}\left((-Y)^{k} \psi_{1} \phi\right) \epsilon_{2-d, d}$. 
Since $\rho$ does not appear on the right side and $\psi_{0}, \psi_{1}$ do not appear on the left, both sides are independent of $\rho, \psi_{0}$ and $\psi_{1}$.

We have proved more than was stated and shall return to this proof later for a more refined statement. $\int_{\mathbf{D}} \phi \epsilon_{n, d}$ turns out to be continuous in $\phi$ as it ranges through a Banach space of $C^{k}$ test functions.

We now turn our attention to the connection between $e_{*}(b, n)$ on $S$ and $\epsilon_{n, d}$ on $\mathrm{D}$. The link is the function $e(b, 1): S \rightarrow \mathrm{D}$. Note that

$$
e_{*}(b, n)=\epsilon_{n, d} \circ e(b, 1) \text {. }
$$

Also the function $r_{t}$ of 3.2 may be expressed as $\rho_{t} \circ e(b, 1)$.

The idea is this: composition with $e(b, 1)$ gives an isometric injection $L^{\infty}(\mathrm{D}) \rightarrow L^{\infty}(S)$. It turns out that this injection is the adjoint of a map $E_{b}$ : $L^{1}(S) \rightarrow L^{1}(\mathrm{D})$. Thus for $f \in L^{1}(S)$ and $\phi \in L^{\infty}(\mathrm{D})$

$$
\int_{S}(\phi \circ e(b, 1)) f=\int_{D} \phi E_{b}(f) .
$$

In particular if $\phi=\rho_{t} \epsilon_{n, d}$ then

$$
\int_{S} r_{t} e_{*}(b, n) f=\int_{\mathrm{D}} \rho_{t} \epsilon_{n, d} E_{b}(f) .
$$

Thus the study of $\lim _{t \rightarrow \infty} \int_{S} r_{t} e_{*}(b, n) f$ is reduced to Lemma 5.1 plus a study of the map $E_{b}$, which we now undertake. The discussion is a long one, partly because we wish to develop a calculus of the map $E_{b}$.

LEMMA 5.2. There exists a unique continuous linear map $E_{b}: L^{1}(S) \rightarrow$ $L^{1}(D)$ of norm 1 such that for all $f \in L^{1}(S)$ and $\phi \in L^{\infty}(S)$

$$
\int_{S}(\phi \circ e(b, 1)) f=\int_{D} \phi E_{b}(f) .
$$

Before beginning the proof we will introduce a bit of machinery that forms part of the calculus of $E_{b}$.

Remark 5.3. Fix $b \in B$. Let $B^{\prime}(b)$ denote the unit sphere in $\{a, b\}^{\perp}$, i.e. $B^{\prime}(b)=\left\{b^{\prime} \in B \mid b^{\prime} \cdot b=0\right\}$. Define a continuous surjection $\Omega_{b}: \mathbf{D} \times B^{\prime}(b) \rightarrow$ $S$ by

$$
\Omega_{b}\left(x+i y, b^{\prime}\right)=x a+y b+\left(1-x^{2}-y^{2}\right)^{1 / 2} b^{\prime}
$$

Then

$$
\begin{aligned}
& e(b, 1)\left(\Omega_{b}\left(z, b^{\prime}\right)\right)=z \quad\left(\left(z, b^{\prime}\right) \in \mathrm{D} \times B^{\prime}(b)\right), \\
& \Omega_{b}\left(e^{i \theta}, b^{\prime}\right)=\cos (\theta) a+\sin (\theta) b \quad(\theta \in[0,2 \pi]) .
\end{aligned}
$$

Let $\mathrm{D}^{\circ}$ denote the interior of $\mathrm{D}$ and $S^{\circ}(b)=e(b, 1)^{-1}\left(\mathrm{D}^{\circ}\right)$. Then 
Since $B^{\prime}(b)$ is a sphere (the unit sphere in $\{a, b\}^{\perp}$ ) it makes sense to speak of its normalized rotation invariant measure. This measure will be assumed.

Lemma 5.4. The map $\Omega_{b}: \mathbf{D} \times B^{\prime}(b) \rightarrow S$ preserves sets of measure zero (where we are assuming the product measure on $\mathbf{D} \times B^{\prime}(b)$ ).

Proof. Let $N \subset \mathrm{D} \times B^{\prime}(b)$ be of measure zero. Write $N=N_{\partial} \cup N_{0}$ where $N_{\partial}=N \cap(\partial \mathrm{D}) \times B^{\prime}(b)$ and $N_{0}=\mathrm{D}^{\circ} \times B^{\prime}(b)$. Then $\Omega_{b}\left(N_{\partial}\right)$ is of measure zero by (5.3.2). Also $\Omega_{b}: D^{\circ} \times B^{\prime}(b) \rightarrow S^{\circ}(b)$ preserves sets of measure zero because it is a diffeomorphism and the measures in question are equivalent (in the sense of absolute continuity) in any coordinate patch to Lebesgue measure. Thus $\Omega_{b}(N)$ is the union of two sets of measure zero.

Proof of 5.2. Fix $b \in B$ and abbreviate $e(b, 1)$ by $e$. We claim first of all that $e: S \rightarrow \mathrm{D}$ has the property

(5.2.2) If $N \subset \mathrm{D}$ has measure zero then $e^{-1}(N) \subset S$ does also.

To see this observe that $e$ may be factored as $p \circ \Omega_{b}^{-1}$ where $p: \mathbf{D} \times B^{\prime}(b)$ $\rightarrow \mathrm{D}$ is the projection. Thus $e^{-1}(N)=\Omega_{b}\left(p^{-1}(N)\right) \cdot p^{-1}(N)$ is of measure zero since the measure on $\mathbf{D} \times B^{\prime}(b)$ is the product measure. Now 5.4 proves (5.2.2).

The rest of the argument rests on (5.2.2). The idea is roughly that $E_{b}$ is like a conditional expectation.

For $f \in L^{1}(S)$ let $\mu_{f}$ denote the Borel measure on $S$ such that $\mu_{f}(A)=$ $\int_{A} f(s) d s$ where $A$ is a Borel set in $S$. (This is the only occasion on which we use a measure different from the normalized rotation invariant one which in the present notation is $\mu_{1} . d \mu_{f}=f d \mu_{1}$.)

Form a new measure $\nu_{f}$ on $\mathbf{D}$ by $\nu_{f}=\mu_{f} \circ e^{-1}$. This is a Borel signed measure with total variation $\leqslant$ that of $\mu_{f}$, i.e., $\leqslant\|f\|_{1}$. Moreover $\nu_{f}$ is absolutely continuous with respect to Lebesgue measure $\lambda$ on D by (5.2.2). Define $E_{b}(f)=$ $d \nu_{f} / d \lambda$, the Radon-Nikodým derivative. $E_{b}$ is linear since the maps $f \rightarrow \mu_{f} \rightarrow$ $v_{f} \rightarrow d \nu_{f} / d \lambda$ are and $\left\|E_{b}(f)\right\|_{1}$ equals the total variation of $v_{f}$ which is $\leqslant$ that of $\mu_{f}$ which is $\|f\|_{1}$. Thus $\left\|E_{b}\right\| \leqslant 1$.

Now let $A \subset \mathrm{D}$ be Borel measurable and let $\phi$ be its characteristic function. Then $\phi \circ e$ is the characteristic function of $e^{-1}(A)$ and

$$
\int_{S}(\phi \circ e) f=\int_{e^{-1}(A)} f=v_{f}(A)=\int_{\mathrm{D}} \phi E_{b}(f) .
$$

Thus (5.2.1) holds in this special case. However since both sides of (5.2.1) are linear and continuous in $\phi \in L^{\infty}$ (D) we have that (5.2.1) holds in general.

(5.2.1) is equivalent to the statement that $E_{b}^{*}: L^{\infty}(\mathrm{D}) \rightarrow L^{\infty}(S)$ is given by $E_{b}^{*}(\phi)=\phi \circ e$. (Note that $\phi \circ e$ is well defined in $L^{\infty}(S)$ by (5.2.2).) This makes 
it clear that $E_{b}$ can be the only map from $L^{1}(S)$ to $L^{1}(D)$ satisfying (5.2.1) because the adjoint uniquely determines the map.

CoRollary 5.5. Given $f \in L^{1}(S)$ and $\psi \in L^{\infty}(\mathrm{D})$ we have $E_{b}((\psi \circ e(b, 1)) f)$ $=\psi E_{b}(f)$.

Proof. For $\phi \in L^{\infty}(\mathrm{D})$ we have (setting $e=e(b, 1)$ )

$$
\int_{D} \phi E_{b}((\psi \circ e) f)=\int_{S}((\phi \psi) \circ e) f=\int_{D} \phi \psi E_{b}(f)
$$

by (5.2.1). Since $\phi$ is arbitrary we are done.

We will need to study the effect of certain group translations on $E_{b}$. Let $K$ denote the subgroup of $G$ (= the full rotation group) which fixes the point $a$. Then $K$ operates transitively on the sphere $B$. Also if $g \in G$ and $f$ is a function on $S$ we write $f^{g}(s)=f(g s)$.

LEMMA 5.6. For $b \in B, k \in K$, and $f \in L^{1}(S)$ we have $E_{b}\left(f^{k}\right)=E_{k b}(f)$, (or equivalently, $\left.E_{k^{-1} b}\left(f^{k}\right)=E_{b}(f)\right)$.

Proof. Define $E_{k, b}(f)=E_{k-1}\left(f^{k}\right)$. It suffices to show that $E_{k, b}$ satisfies (5.2.1). Choose any $\phi \in L^{\infty}(\mathrm{D})$. Then by (5.2.1)

$$
\int_{\mathrm{D}} \phi E_{k, b}(f)=\int_{\mathrm{D}} \phi E_{k^{-1} b}\left(f^{k}\right)=\int_{S}\left(\phi \circ e\left(k^{-1} b, 1\right)\right) f^{k} .
$$

Note that

$$
\begin{aligned}
e\left(k^{-1} b, 1\right)(s) & =a \cdot s+i k^{-1} b \cdot s=a \cdot s+i b \cdot k s \\
& =e(b, 1)(k s)=e(b, 1)^{k}(s) .
\end{aligned}
$$

Thus

$$
\phi \circ e\left(k^{-1} b, 1\right)=(\phi \circ e(b, 1))^{k}
$$

Thus

$$
\int_{\mathrm{D}} \phi E_{k, b}(f)=\int_{S}(\phi \circ e(b, 1))^{k} f^{k}=\int_{S}(\phi \circ e(b, 1)) f .
$$

CoRollary 5.7. If $M_{b}$ denotes the subgroup of $K$ which also fixes the point $b$ then for $m \in M_{b}, E_{b}\left(f^{m}\right)=E_{b}(f)$.

Corollary 5.8. Normalize Haar measure on $M_{b}$ so that $\int_{M_{b}} 1=1$. Then for $f \in L^{1}(S), E_{b}\left(\int_{M_{b}} f^{m} d m\right)=E_{b}(f)$.

Proof. $m \rightarrow f^{m}$ is continuous from $M_{b}$ to the Banach space $L^{1}(S)$ so $\int_{M_{b}} f^{m} d m$ is well defined. Since $E_{b}$ is continuous from $L^{1}(S)$ to $L^{1}(D)$ we have 


$$
E_{b}\left(\int_{M_{b}} f^{m} d m\right)=\int_{M_{b}} E_{b}\left(f^{m}\right) d m=\int_{M_{b}} E_{b}(f) d m
$$

by the previous corollary. Now on the right we have the integral of a constant which is again that constant.

Definition 5.9. For $f \in L^{1}(S)$ we define $A_{b}(f) \in L^{1}(S)$ by

$$
A_{b}(f)=\int_{M_{b}} f^{m} d m \text {. }
$$

Observe that $A_{b}(f)$ is again in $L^{1}(S)$ by the Fubini theorem and that $A_{b}(f)$ is constant on the orbits in $S$ of the group $M_{b}$. Since these orbits are precisely the sets $e(b, 1)^{-1}(z)$ as $z \in D$ there exists a measurable function $\alpha_{b}(f)$ on $\mathbf{D}$ such that

$$
\alpha_{b}(f) \circ e(b, 1)=A_{b}(f) \text {. }
$$

LEMMA 5.10. If $f \in L^{\infty}(S)$ then $\alpha_{b}(f) \in L^{\infty}(\mathrm{D})$ with $\left\|\alpha_{b}(f)\right\|_{\infty} \leqslant\|f\|_{\infty}$. Moreover

$$
E_{b}(f)=\alpha_{b}(f) E_{b}(1)
$$

Proof. $A_{b}(f)(s)$ is the average of $f$ over the orbit $M_{b} s$ so $\left\|A_{b}(f)\right\|_{\infty} \leqslant$ $\|f\|_{\infty}$. Clearly $\left\|\alpha_{b}(f)\right\|_{\infty}=\left\|A_{b}(f)\right\|_{\infty}$ so the first assertion is clear.

Now by $5.8,5.9$ and 5.5 (in that order)

$$
E_{b}(f)=E_{b}\left(A_{b}(f)\right)=E_{b}\left(\left(\alpha_{b}(f) \circ e(b, 1)\right) 1\right)=\alpha_{b}(f) E_{b}(1) .
$$

This lemma gives an often useful method of computing $E_{b}$ from $A_{b}$ and the function $E_{b}(1)$ which is given explicitly in Lemma 5.12. A second method of computing $\alpha_{b}(f)$ is obtained from $\Omega_{b}$ in

Lemma 5.11. For $f \in L^{\infty}(S)$

$$
\alpha_{b}(f)(z)=\int_{B^{\prime}(b)} f\left(\Omega_{b}\left(z, b^{\prime}\right)\right) d b^{\prime} .
$$

Proof. It is easily seen that for $m \in M_{b}, b^{\prime \prime} \in B^{\prime}(b)$ we have $m \Omega_{b}\left(z, b^{\prime \prime}\right)$ $=\Omega_{b}\left(z, m b^{\prime \prime}\right)$. For almost all $z \in \mathrm{D}$

$$
\begin{aligned}
A_{b}(f)\left(\Omega_{b}\left(z, b^{\prime \prime}\right)\right) & =\int_{M_{b}} f\left(m \Omega_{b}\left(z, b^{\prime \prime}\right)\right) d m \\
& =\int_{M_{b}} f\left(\Omega_{b}\left(z, m b^{\prime \prime}\right)\right) d m=\int_{B^{\prime}(b)} f\left(\Omega_{b}\left(z, b^{\prime}\right)\right) d b^{\prime} .
\end{aligned}
$$

The last equality on the right follows from the fact that for any $\phi \in L^{1}\left(B^{\prime}(b)\right)$ $\int_{B^{\prime}(b)} \phi\left(b^{\prime}\right) d b^{\prime}=\int_{M_{b}} \phi\left(m b^{\prime \prime}\right) d m$ since both sides express an $M_{b^{-}}$-invariant normalized integral.

LEMMA 5.12. $E_{b}(1)(z)=(d-1)\left(1-|z|^{2}\right)^{(d-3) / 2} / 2 \pi(z \in \mathrm{D})$. 
Proof. Let $f \in L^{1}(S)$ and $\lambda$ denote Lebesgue measure on D. Then

$$
\int_{S} f=\int_{\mathrm{D} \times B^{\prime}(b)} f\left(\Omega_{b}\left(z, b^{\prime}\right)\right) E_{b}(1)(z) d \lambda(z) d b^{\prime} .
$$

To prove (5.12.1) multiply both sides of the equation in 5.11 by $E_{b}(1)(z)$ and integrate over $\mathbf{D}$. The right side coincides with the right side of $(5.12 .1)$ and the left side is

$$
\int_{\mathrm{D}} E_{b}(1)(z) \alpha_{b}(f)(z) d \lambda(z)=\int_{\mathrm{D}} E_{b}(f)=\int_{S} f
$$

by (5.2.1). This proves (5.12.1).

The message of (5.12.1) is that the map $\Omega_{b}: \mathrm{D} \times B^{\prime}(b) \rightarrow S$ pulls the measure on $S$ back to a function $J$ times the product measure on $\mathrm{D} \times B^{\prime}(b)$ and $J$ is given by $J\left(z, b^{\prime}\right)=E_{b}(1)(z)$.

The point is that we can compute $J$ explicitly. Although this is routine we include a sketch of how this can be done expeditiously.

Let $W=\{a, b\}^{\perp} . B^{\prime}(b)$ is the unit sphere in $W$. Extend $\Omega_{b}$ to a map $\bar{\Omega}_{b}: \mathbf{D} \times W \rightarrow \mathbf{R}^{d+1}$ by

$$
\bar{\Omega}_{b}(x+i y, w)=\|w\|(x a+y b)+\left(1-x^{2}-y^{2}\right)^{1 / 2} w .
$$

The measures on $\mathbf{D} \times B^{\prime}(b)$ and $S$ are connected to Lebesgue measure on $\mathbf{D} \times W$ and $\mathbf{R}^{d+1}$ respectively by introducing polar coordinates. It is an easy matter to compute the Jacobian of $\bar{\Omega}_{b}$ and relate this to $J$ through polar coordinates. The result is that $J\left(z, b^{\prime}\right)=c\left(1-|z|^{2}\right)^{(d-3) / 2}$ where $c$ is a constant. The value of $c$ is found from

$$
1=\int_{S} 1=\int_{D} E_{b}(1)=c \int_{0}^{2 \pi} \int_{0}^{1}\left(1-r^{2}\right)^{(d-3) / 2} r d r d \theta .
$$

We know enough about $E_{b}$ at this point to begin a study of its smoothness preserving qualities. Picture a neighborhood $U$ of 0 in $\mathbf{D}$ which is so small that the closure of $U$ is in $\mathrm{D}^{\circ}$. Imagine an open set $V$ in $S$ which contains $e(b, 1)^{-1}(U)$. If a function $f \in L^{1}(S)$ has uniformly continuous derivatives on $V$ up to order $n+d-2$ then we will show below that the same is true of $E_{b}(f)$ on $U$. Consequently, we may compute $\int_{S} f e_{*}(b, n)$ from $\int_{D} E_{b}(f) \epsilon_{n, d}$ by 5.2 (see the discussion preceding 5.2). For a while now our concern will be to make the suggestion of this paragraph precise.

DEFinition 5.13. Let $X$ be a $C^{\infty}$ manifold with a fixed finite measure. Let $U$ be an open subset of $X$ with compact closure. Let $m$ be a positive integer. $L C^{m}(X, U)$ will denote the space of $L^{1}$ functions on $X$ having uniformly continuous derivatives up to order $m$ on $U$. By this is meant the following:

If $f \in L C^{m}(X, U)$ then $\int_{X}|f|<\infty$ and if $D$ is any $C^{\infty}$-coefficient differential 
operator defined in a neighborhood of $U$ and of order $\leqslant m$ then $D(f \mid U)$ is defined and extends to a continuous function on $\bar{U}$.

REMARK 5.14. $L C^{m}(X, U)$ is a topological vector space with convergence defined as convergence in $L^{1}(X)$ plus uniform convergence on $U$ in all derivatives up to order $m$. With this topology $L C^{m}(X, U)$ is normable and complete. Also, if $g: X \rightarrow X$ is a measure preserving $C^{\infty}$ diffeomorphism with $g(U)=U$ then $f \rightarrow f^{g}$ is a continuous automorphism of $L C^{m}(X, U)$.

Definition 5.15. Fix a number $l: 0<l<1$ for the duration of the paper. Denote

$$
\begin{array}{ll}
l \mathbf{D}^{\circ}=\left\{l z \mid z \in \mathrm{D}^{\circ}\right\}=\{z \in \mathrm{D}|| z \mid<l\}, & \mathfrak{D}^{m}=L C^{m}\left(\mathrm{D}^{\circ}, l \mathrm{D}^{\circ}\right) . \\
S[l]=\{s \in S|| s \cdot a \mid>l\}, & S^{m}=L C^{m}(S, S[l]),
\end{array}
$$

Lemma 5.16. $E_{b}$ maps $\mathfrak{S}^{m}$ into $\mathfrak{D}^{m}$ and is a continuous linear operator between these spaces.

Proof. We already have that $E_{b}: L^{1}(S) \rightarrow L^{1}(\mathrm{D})$ is continuous. We have to consider $E_{b}(f)$ on $l \mathrm{D}^{\circ}$ for $f \in L C^{m}(S, S[l])$. Since $E_{b}(f)=\alpha_{b}(f) E_{b}(1)$ and $E_{b}(1)$ is $C^{\infty}$ in $D^{\circ}$ it suffices to consider $\alpha_{b}(f) \mid D^{\circ}$.

Now by $5.11, \alpha_{b}(f)(z)=\int_{B^{\prime}(b)} f\left(\Omega_{b}\left(z, b^{\prime}\right)\right) d b^{\prime}$.

If $f \in L C^{m}(S, S[l])$ then $f \circ \Omega_{b}$ is of class $C^{m}$ on $l D^{\circ} \times B^{\prime}(b)$ with uniformly continuous derivatives up to order $m$ (i.e. they extend continuously to ID $\left.\times B^{\prime}(b)\right)$. Consequently, $\alpha_{b}(f)$ is also uniformly continuous in all derivatives up to order $m$ on $l D^{\circ}$ and we may bound the sup norm of a derivative $(\partial / \partial x)^{j}(\partial / \partial y)^{k} \alpha_{b}(f)(x+i y)$ by the sup norm of the same operator applied to $f \circ \Omega_{b}$.

Lemma 5.17. In the notation of Lemma 5.1 and Definition 5.15 the linear functionals on $\mathfrak{D}^{n+d-2}$ given by $\phi \rightarrow \int_{D} \phi \rho_{t} \epsilon_{n, d}\left(\phi \in \mathfrak{D}^{n+d-2}\right)$ are continuous and converge uniformly as $t \rightarrow \infty$ to a continuous linear functional as in 5.1.

Proof. A reexamination of the proof of 5.1 is sufficient proof. Take the open set $U$ to be $l \mathrm{D}^{\circ}$. The convergence of $\int_{\mathrm{D}} \rho_{t} \phi \psi_{0} \epsilon_{n, d}$ is uniform in $\phi \in L^{1}$ (D) and the convergence of $\int_{\mathrm{D}} \rho_{t} \phi \psi_{1} \epsilon_{n, d}=(k !)^{-1} \int_{\mathrm{D}} \rho_{t}\left((-Y)^{n+d-2} \psi_{1} \phi\right) \epsilon_{2-d, d}$ is uniform in $\psi_{1} \phi \in C_{c}^{n+d-2}\left(\mathrm{DD}^{\circ}\right)$.

LEMMA 5.18. In the notation of 3.1 and 5.15 , if $f \in S^{n+d-2}$ then $F_{t} f(b, n)$ exists and is continuous in $f \in S^{n+d-2}$. As $t \rightarrow \infty$ this continuous linear functional converges uniformly in $f$ to $F_{*} f(b, n)$.

Proof. By definition of $F_{t}$ and by 5.3 


$$
F_{t} f(b, n)=\int_{S}\left(\left(\rho_{t} \epsilon_{n, d}\right) \circ e(b, 1)\right) f=\int_{\mathrm{D}} \rho_{t} \epsilon_{n, d} E_{b}(f) .
$$

Now the result follows by combining 5.16 and 5.17 .

Lemma 5.19. Fix $f \in \mathfrak{S}^{m}, m \geqslant 0$. Then the map $k \rightarrow f^{k}$ from $K$ to $\mathfrak{S}^{m}$ is continuous. In particular $\left\{f^{k} \mid k \in K\right\}$ is compact in $\mathfrak{S}^{m}$.

Proof. The continuity of $k \rightarrow f^{k}$ into $L^{1}(S)$ is a well-known consequence of the fact that the uniformly continuous functions on $S$ are dense in $L^{1}(S)$.

The continuity of $k \rightarrow f^{k} \mid S[l]$ into $C^{m}(S[l])$ follows from the uniform continuity of derivatives of $f$ up to order $m$ on the $K$-invariant set $S[l]$.

The continuity of $k \rightarrow f^{k}$ into $\mathfrak{S}^{m}$ is a combination of these two kinds of continuity.

LEMMA 5.20. For $f \in \mathfrak{S}^{n+d-2}$ we have

$$
\begin{gathered}
F_{t} f(k b, n)=F_{t} f^{k}(b, n) \quad(k \in K, b \in B), \\
\lim _{t \rightarrow \infty} F_{t} f(b, n)=F_{*} f(b, n) \text { uniformly in } b \in B, \\
F_{*} f(k b, n)=F_{*} f^{k}(b, n) \quad(k \in K, b \in B) .
\end{gathered}
$$

Proof. (5.20.1) follows from Lemma 5.5 and the formula $F_{t} f(b, n)=$ $\int_{\mathrm{D}} E_{b}(f) \rho_{t} \epsilon_{n, d}$.

(5.20.2) is a corollary of Lemmas 5.18 and 5.19.

(5.20.3) is immediate from (5.20.1) and (5.20.2).

Lemmas 5.18 and 5.20 together prove Lemma 3.3. Indeed 5.18 and 5.20 constitute a statement that is more refined than 3.3 in a useful way. For one thing the exact degree of differentiability of $f$ (namely $n+d-2$ ) used in the proof is displayed. For another thing it is clear that this differentiability is needed only in $S[l]$ and $l$ could be chosen as close to 0 as desired.

The formulae (5.20.1) and (5.20.3) are of great interest. For one thing they show that if $f$ is a polynomial of degree $m$ then $F_{*} f(b, n)$ is the restriction to $B$ of a polynomial of degree $\leqslant m$. Also they show that if $f \in D$ then $F_{t} f(b, n)$ is $C^{\infty}$ in $b$ and converges uniformly in all derivatives to $F_{*} f(b, n)$ ( $n$ fixed, $b$ variable in $B$ ).

We can do even better than that. Suppose $f \in C(S)$ and is of class $C^{m}$ in "the direction parallel to the equator $B$ " in the following sense: $k \rightarrow f^{k}$ is of class $C^{m}$ from the Lie grour $K$ to the Banach space $C(S)$. Then $E_{b}(f)(x+i y)$ would be of class $C^{m}$ in $y$ (for $z=x+i y$ in $\mathrm{D}^{\circ}$ ) and if $m=n+d-2$ we may 
apply a refined version of the proof of Lemma 5.1 to establish a result like 5.18. This raises the interesting possibility of defining $F_{*} f(b, n)$ for $f \in C(S)$ as a distribution of $B$ of degree $n+d-2$. That is, if $\phi \in C^{n+d-2}(B)$ then $\int_{B} F_{t} f(b, n) \phi(b) d b$ converges as $t \rightarrow \infty$ to a value $\int_{B} F_{*} f(b, n) \phi(b) d b$ which is linear and continuous in $\phi \in C^{n+d-2}$. The idea here is

$$
\begin{aligned}
\int_{B} F_{t} f(b, n) \phi(b) d b & =\int_{K} F_{t} f\left(k b_{1}, n\right) \phi\left(k b_{1}\right) d k \\
& =F_{t}\left(\int_{K} \phi\left(k b_{1}\right) f^{k} d k\right)\left(b_{1}, n\right)
\end{aligned}
$$

and $\int_{K} \phi\left(k b_{1}\right) f^{k} d k$ is of class $C^{n+d-2}$ "in the direction parallel to the equator $B$ " on $S$. This is not a proof but it indicates an important potential for extending the results of this section. Let us then state

Conjecture 5.21. For $f \in C(S)$ we may define $F_{*} f(b, n)$ as a distribution on $B$ of order $n+d-2$. Moreover for $\phi \in C^{n+d-2}(B)$,

$$
\lim _{t \rightarrow \infty} \int_{B} \phi(b) F_{t} f(b, n) d b=\int_{B} \phi(b) F_{*} f(b, n) d b
$$

and postpone a full proof to another paper.

6. Proof of the main results. We will prove the results stated in $\S 3$. The first is Lemma 3.3. At the end of $\S 5$ we observed that 3.3 follows from 5.18 and 5.20. Next we have Theorem 3.4.

Proof of 3.4. Recall that by (2.3)

$$
f_{n}^{\prime}\left(s_{1}\right)=\operatorname{dim}\left(H_{n}\right) \int_{S} f^{\prime}(s) P_{n}\left(s_{1} \cdot s\right) d s
$$

Thus it suffices to prove that for $s_{1} \in S-B$

$$
\int_{S} f^{\prime}(s) P_{n}\left(s_{1} \cdot s\right) d s=\int_{B} e_{*}(b, n)\left(s_{1}\right) \int_{S} f^{\prime}(s) e(b, n)(s) d s d b .
$$

But by the Key Lemma $3.9, P_{n}\left(s_{1} \cdot s\right)=\int_{B} e_{*}(b, n)\left(s_{1}\right) e(b, n)(s) d b$. Essentially then this result amounts to an interchange of the order of integration except that $f^{\prime}$ is a distribution. (However if $f^{\prime} \in L^{1}(S)$ we are done at this point by the Fubini theorem applied to the integral over $S \times B$ of $(s, b) \rightarrow f^{\prime}(s) e_{*}(b, n)\left(s_{1}\right)$. $e(b, n)(s)\left(s_{1}\right.$ fixed).) To cover the general case of $f^{\prime} \in D^{\prime}$ observe that $b \rightarrow$ $e(b, n)$ is continuous from $B$ to $D$. Also, for fixed $s_{1} \in S-B, e_{*}(b, n)\left(s_{1}\right)$ is continuous in $b$, so $b \rightarrow e_{*}(b, n)\left(s_{1}\right) e(b, n)$ is continuous from $B$ to $D$. Thus the $D$ valued Riemann integral $\int_{B} e_{*}(b, n)\left(s_{1}\right) e(b, n) d b$ converges in $D$ to $S \rightarrow P_{n}\left(s \cdot s_{1}\right)$. Now if we apply the continuous linear functional $f^{\prime}$ we get

$$
\int_{S} f^{\prime} \int_{B} e^{*}(b, n)\left(s_{1}\right) e(b, n) d b=\int_{B} e_{*}(b, n)\left(s_{1}\right)\left(\int_{S} f^{\prime} e(b, n)\right) d b .
$$


The proof of Corollary 3.5 is immediate from the Hilbert space direct sum decomposition of $f \in H$ into the sum of terms $f_{n} \in H_{n}$ plus 3.4 which gives

$$
f_{n}\left(s_{1}\right)=\operatorname{dim}\left(H_{n}\right) \int_{B} F f(b, n) e_{*}(b, n)\left(s_{1}\right) d b
$$

almost everywhere. The uniform convergence statement is a corollary of the theorem stated at the end of $\S 2$.

Theorem 3.6 is the one which calls on $\S 5$.

Proof of 3.6. Recall the function $r_{t}$ and $F_{t} f$ as defined in 3.2. By the Lebesgue dominated convergence theorem

$$
\begin{aligned}
\int_{S} f\left(s_{1}\right) P_{n}\left(s_{1} \cdot s\right) d s_{1} & =\lim _{t \rightarrow \infty} \int_{S} r_{t}\left(s_{1}\right) f\left(s_{1}\right) P_{n}\left(s_{1} \cdot s\right) d s_{1} \\
& =\lim _{t \rightarrow \infty} \int_{S} \int_{B} r_{t}\left(s_{1}\right) f\left(s_{1}\right) e_{*}(b, n)\left(s_{1}\right) e(b, n)(s) d b d s_{1} .
\end{aligned}
$$

Since $r_{t} e_{*}(b, n)$ is smooth we can reverse the order of integration and obtain

$$
\begin{gathered}
\lim _{t \rightarrow \infty} \int_{B} \int_{S} r_{t}\left(s_{1}\right) f\left(s_{1}\right) e_{*}(b, n)\left(s_{1}\right) d s_{1} e(b, n)(s) d b \\
=\lim _{t \rightarrow \infty} \int_{B} F_{t} f(b, n) e(b, n)(s) d b .
\end{gathered}
$$

By Lemma 3.3 $F_{t} f(b, n)$ converges to $F_{*} f(b, n)$ uniformly on $B$ so the above limit becomes $\int_{B} F_{*} f(b, n) e(b, n)(s) d b$. Thus

$$
f_{n}(s)=\operatorname{dim}\left(H_{n}\right) \int_{S} f\left(s_{1}\right) P_{n}\left(s \cdot s_{1}\right) d s_{1}=\operatorname{dim}\left(H_{n}\right) \int_{B} F_{*} f(b, n) e(b, n) d b .
$$

Corollary 3.7 follows from 3.6 and the theorem at the end of $\S 2$.

The idea of Theorem 3.8 is a combination of the ideas in 3.4 and 3.6.

Proof of 3.8. Since $\int_{S} f f^{\prime}=\Sigma \int_{S} f_{n} f^{\prime}$ (sum over $n \in N$ ) we have only to show that

$$
\int_{S} f_{n} f_{n}^{\prime}=\operatorname{dim}\left(H_{n}\right) \int_{B} F_{*} f(b, n) F f^{\prime}(b, n) d b .
$$

By Theorem 3.6 we have $f_{n}=\operatorname{dim}\left(H_{n}\right) \int_{B} F_{*} f(b, n) e(b, n) \cdot d b$ where we may regard this as the Riemann integral on the continuous $D$-valued function on $B$ namely $b \rightarrow F_{*} f(b, n) e(b, n)$. By an argument similar to the one in 3.4 we have

$$
\begin{aligned}
\int_{S} f^{\prime} f_{n} & =\operatorname{dim}\left(H_{n}\right) \int_{S}\left(f^{\prime} \int_{B} F_{*} f(b, n) e(b, n) d b\right) \\
& =\int_{B} F_{*} f(b, n)\left(\int_{S} f^{\prime} e(b, n)\right) d b=\int_{B} F_{*} f(b, n) F f^{\prime}(b, n) d b .
\end{aligned}
$$

Appendix 1. This appendix is a brief for the proposition that $e(b, n)$ is to 
$S$ as characters are to abelian groups. We have three observations to make in evidence of this proposition.

FIRST OBSERVATION. In the case $d=1, S$ has the structure of an abelian group. In this case the functions $e(b, n)$ are precisely the characters. This case is exceptional in that the functions $e_{*}(b, n)$ are nonsingular and are in fact also characters. Our theory in this case is precisely the theory of Fourier series.

SECOND OBSERVATION. Imagine that $S$ is "inflated" until a neighborhood of the north pole closely approximates Euclidean space of the same dimension $d$. Then the class of functions $e(b, n)$ approximates the class of characters. (We will make this precise in a moment.)

THIRD OBSERVATION. The functions $e(b, n)$ are eigenfunctions for a $d$ dimensional Lie algebra of complex infinitesimal motions of $S$; they are characterized by this Lie algebra (which depends on $b$ ) and the normalizing condition that $e(b, n)(a)=1$.

To clarify our second and third observations let $V$ denote $\{a\}^{\perp}$ in $\mathbf{R}^{d+1}$. Then $S$ and $V$ have the same dimension $d$. We are claiming a similarity between harmonic analysis on $S$ and on $V$. Note that $B$ is the unit sphere in $V$. A character on $V$ has the form

$$
v \rightarrow \epsilon(b, r)(v)=(\exp (i b \cdot v))^{r} \quad(b \in B, r \geqslant 0, v \in V) .
$$

To make our second observation precise first imagine $S$ "inflated" by a large number $N$ to a sphere $N S$ of radius $N$ but concentric with $S$. There is a natural bijection

$$
S \leftrightarrow N S, \quad s \leftrightarrow N s \quad(s \in S)
$$

which lets us lift our theory wholesale to $N S$. In particular we write $e(b, n)(N s)=$ $e(b, n)(s)$.

Now consider the map $\gamma_{N}$ of $V$ to $N S$ given by

$$
\gamma_{N}(v)=\cos (|v| / N) N a+\sin (|v| / N)(N /|v|) v .
$$

$\gamma_{N}$ maps rays from the origin in $V$ to geodesics from $N a$ in $N S$ in a way that preserves arc length. Also $\gamma_{N}$ approximates the map $v \rightarrow N a+v$ of $V$ to the tangent space $N a+V$ to $N S$ at $N a$, at least for small $v$. This approximation is what is meant by saying that $N S$ approximates the Euclidean space $\mathrm{Na}+\mathrm{V}$, or by proxy, $V$.

Now consider $e(b, n)$ on $N S$ composed with $\gamma_{N}$ :

$$
e(b, n)\left(\gamma_{N}(v)\right)=(\cos (|v| / N)+i(b \cdot v)(|v|) \sin (|v| / N))^{n} .
$$

If we fix $r \geqslant 0$ and choose $N=n / r$ very large compared to $|v|$ then the above 
expression is approximately $(1+i(b \cdot v) / N)^{r N} \approx \epsilon(b, r)(v)$. If $v$ is a multiple of $b$ then we have a precise equality:

$$
e(b, n)\left(\gamma_{N}(v)\right)=\epsilon(b, r)(v) .
$$

There are three comments worth making about this asymptotic relation between $e(b, n)$ and $\epsilon(b, r)$. The first comment is that in case $d=1$ this is the method used by Fourier to go from Fourier series on the circle to the Fourier integrals on the line. In this connection note that when $d=1 v$ is always a multiple of $b$ so $e(b, n) \circ \gamma_{N}=\epsilon(b, r)$. This is just the lifting of a character from the circle to $\mathrm{R}$ by the covering map $\gamma_{N}$.

The second comment is that the asymptotic relation between $e(b, n)$ and $\epsilon(b, r)$ explains various classical formulae expressing Bessel coefficients as limits of associated Legendre functions. (See, for example, Whittaker and Watson, §17.4.) Take $d=2$. Then $B$ is the circle. The limit relation

$$
\lim _{N \rightarrow \infty} e(b, n)\left(\gamma_{n / r}(v)\right)=\epsilon(b, r) \quad(n=r N)
$$

implies a convergence of

$$
P_{n}(\cos (|v| r / n))=\int_{B} e(b, n)\left(\gamma_{n / r}(v)\right) d b
$$

$($ as $n \rightarrow \infty)$ to

$$
J \circ(r|v|)=\int_{B} \epsilon(b, r)(v) d b .
$$

A similar formula holds for the higher Bessel coefficients if we introduce a character of the circle group $B$ when we integrate over $B$. If $d>2$ this method gives formulae which are new to the author if not to the world.

The third comment is that this asymptotic relationship suggests a general principle: Given a phenomenon in harmonic analysis on $V$ which is invariant under dilation: $v \rightarrow t v(t>0)$, look for a corresponding phenomenon in harmonic analysis on $S$ which has the phenomenon on $V$ as a limiting case.

Now to expand upon our third observation: Fix $b \in B$. We associate with every $v \in V$ a complex vector field $z_{v}$ on $S$ by

$$
-i z_{v}(s)=((a+i b) \cdot s) v-(v \cdot s)(a+i b) .
$$

$z_{v}$ extends by complex linearity to a skew-symmetric linear endomorphism of $\mathbf{C}^{d+1}$. The set $\left\{z_{v} \mid v \in V\right\}$ is a real Lie subalgebra of $\operatorname{End}\left(C^{d+1}\right)$ with

$$
\left[z_{u}, z_{v}\right]=z_{w}, \quad w=z_{u}(v)-z_{v}(u) .
$$

Note also that $-i z_{v}(a)=v$. Thus this set of vector fields is linearly independent 
and spans the complex tangent space to $S$ at $a$. (In fact, this will occur at every point of $S$ except for the zero set of $e(b, 1)$.)

Now if we regard the vector field $z_{v}$ as a first order complex coefficient differential operator on $S$ by

$$
\left(z_{v} f\right)(s)=(d / d t) f\left(\exp \left(-t z_{v}\right) s\right)_{\mid t=0}
$$

then

$$
z_{v} e(b, n)=n(v \cdot b) e(b, n) .
$$

This formula is analogous to $-i D_{v} \epsilon(b, r)=r(v \cdot b) \epsilon(b, r)$ where $D_{v}$ denotes directional derivative on $V$ in the direction $v$. The main difference in the case of the sphere is the dependence on $b \in B$. Whereas for $V$ one Lie algebra of vector fields (the infinitesimal translations) serves to define all characters, on the sphere there is a family of Lie algebras, one for each $b$, and a corresponding semigroup of eigenfunctions $e(b, n)(n=0,1, \ldots)$.

Appendix 2. Calculations with $F$ and $F_{*}$. Our purpose here is twofold. We wish to show by example that it is possible to make explicit calculations of $F$ and $F_{*}$. Secondly, we wish to give some general formulae and techniques that will aid further calculations.

This appendix is divided into paragraphs numbered (1)-(8).

(1) We will vary $d$ in this appendix so it is necessary to modify some of our notation thus: where we have written $P_{n}, H_{n}$ and $H_{n}$ we now write $P_{n, d}, H_{n, d}$ and $H_{n, d} \cdot \mathbf{R}^{d}$ is identified with $a^{\perp}$ in $\mathbf{R}^{d+1}$. Thus $B$ is the unit sphere in $\mathbf{R}^{d}$. $H_{n, d-1}$ will be regarded as a space of functions on $B$.

Let $P_{n}(B)$ denote the space of polynomials on $B$ of degree at most $n$. Then $P_{n}(B)=\Sigma_{j=0}^{n} H_{j, d-1}$.

Recall from $\$ 2$ that

$$
\begin{aligned}
\operatorname{dim}\left(H_{n, d}\right) & =(n+d) ! /(n ! d !)-(n+d-2) ! / d !(n-2) ! \\
& =(n+d-1) ! / n !(d-1) !+(n+d-2) ! /(n-1) !(d-1) ! \\
& =(2 n+d-1)((n+d-2) ! / n !(d-1) !) .
\end{aligned}
$$

(These formulae hold for $n \geqslant 2$ and $d \geqslant 1$. We also have $\operatorname{dim}\left(H_{1, d}\right)=d+1$ and $\operatorname{dim}\left(H_{0, d}\right)=1$.)

As a consequence of

$$
\operatorname{dim}\left(H_{j, d-1}\right)=(j+d-1) ! / j !(d-1) !-(j+d-3) ! /(j-2) !(d-1) !
$$

we have 


$$
\operatorname{dim} P_{n}(B)=\sum_{j=0}^{n} \operatorname{dim}\left(H_{j, d-1}\right)=\operatorname{dim}\left(H_{n, d}\right) \text {. }
$$

We will be concerned with specific linear isomorphisms between $P_{n}(B)$ and $H_{n, d}$.

(2) Let $R \in H_{j, d-1}$ and define a function $f_{R}$ on $S$ by

$$
f_{R}(\cos \theta a+\sin \theta b)=(\sin \theta)^{j} P_{n-j, d+2 j}(\cos \theta) R(b) .
$$

Then $f_{R} \in H_{n, d}$ and the map $R \rightarrow f_{R}$ is an injection of $H_{j, d-1}$ into $H_{n, d}$. (See [1, p. 239].)

The map $R \rightarrow f_{R}$ is obviously $K$-equivariant, i.e., if $k$ is a rotation leaving fixed the point $a(k \in K)$ then $f_{R} k=\left(f_{R}\right)^{k}$. An immediate consequence of this fact and the dimension count in (1) is that $H_{n, d}$ is isomorphic to $\Sigma_{j=0}^{n} H_{j, d-1}$ as a $K$-module.

Now suppose $\Phi: H_{n, d} \rightarrow L^{2}(B)$ were any $K$-equivariant map. Then the image of $\Phi$ in $L^{2}(B)$ is $K$-isomorphic to a subspace of $\Sigma_{0}^{n} H_{j, d-1}$ and therefore must lie within $\Sigma_{0}^{n} H_{j, d-1}$.

(3) $F_{*}$ and $F$ are both $K$-equivariant. For $F_{*}$ this is shown in Lemma 5.20. For $F$ it is a simple consequence of the $K$-invariance of $\int_{S}$ and the fact that $e(b, n)(k s)=e\left(k^{-1} b, n\right)(s)$. From (2) it follows that for any $f \in H_{n, d}$ the functions of $b: F f(b, m)$ and $F_{*} f(b, m)(m \geqslant 0$ an integer $)$ are in $P_{n}(B)$.

In more detail, consider $R \in H_{j, d-1}$ and $f_{R}$ as in (2). Then the maps $R \rightarrow f_{R} \rightarrow F f_{R}(b, m), R \rightarrow f_{R} \rightarrow F_{*} f_{R}(b, m)$, are $K$-equivariant. Thus by Schur's lemma and the $K$-irreducibility of $H_{j, d-1}$ there are numbers $\phi(n, j, m, d)$ and $\phi_{*}(n, j, m, d)$ such that

$$
F f_{R}(b, m)=\phi(n, j, m, d) R(b), \quad F_{*} f_{R}(b, m)=\phi_{*}(n, j, m, d) R(b) .
$$

One of our objectives in this appendix is to compute $\phi$ and $\phi_{*}$ explicitly.

(4) From the Parseval formula (Theorem 3.8) we have (with $f_{R}$ as in (2)) that

$$
\begin{aligned}
\int_{S}\left(f_{R}\right)^{2} & =\operatorname{dim}\left(H_{n, d}\right) \int_{B} F f_{R}(b, n) F_{*} f_{R}(b, n) d b \\
& =\operatorname{dim}\left(H_{n, d}\right) \phi(n, j, n, d) \phi_{*}(n, j, n, d) \int_{B} R^{2} .
\end{aligned}
$$

On the other hand, we can compute the integral $\int_{S} g$ of any function $g$ on $S$ as

$$
c_{d} \int_{0}^{\pi} \int_{B} g(\cos \phi a+\sin \phi b)(\sin \phi)^{d-1} d b d \phi
$$

where the constant $c_{d}$ is chosen so that $\int_{S} 1=1$, i.e. $1=c_{d} \int_{0}^{\pi}(\sin \phi)^{d-1} d \phi$.

$$
c_{d}=\Gamma((d+1) / 2) / \Gamma(d / 2) \Gamma(1 / 2)=\Gamma(d) / 2^{d-1}(\Gamma(d / 2))^{2} .
$$


From the definition of $f_{R}$

$$
\int_{S} f_{R}^{2}=c_{d} \int_{0}^{\pi}\left[P_{n-j, 2 j+d}(\cos \phi)\right]^{2}(\sin \phi)^{2 j+d-1} d \phi \int_{B} R^{2} .
$$

We may compute the integral over $[0, \pi]$ by exploiting the fact that

$$
c_{d} \int_{0}^{\pi}\left[P_{n, d}(\cos \phi)\right]^{2}(\sin \phi)^{d-1} d \phi=\int_{S}\left(P_{n, d}(s \cdot a)\right)^{2} d s=\left(\operatorname{dim}\left(H_{n, d}\right)\right)^{-1}
$$

(see (2.3) of $\S 2$ and in (2.3) take $f(s)=f_{n}(s)=P_{n}(s \cdot a)$ and $\left.s_{1}=a\right)$. If we replace $n$ by $n-j$ and $d$ by $d+2 j$ we obtain

$$
c_{d+2 j} \int_{0}^{\pi}\left[P_{n-j, 2 j+d}(\cos \phi)\right]^{2}(\sin \phi)^{2 j+d-1} d \phi=\left(\operatorname{dim}\left(H_{n-j, 2 j+d}\right)\right)^{-1} .
$$

Thus

$$
\begin{array}{r}
\operatorname{dim}\left(H_{n, d}\right) \phi(n, j, n, d) \phi_{*}(n, j, n, d) \int_{B} R^{2}=\int_{S}\left(f_{R}\right)^{2} \\
=\left(c_{d} / c_{d+2 j}\right)\left(\operatorname{dim}\left(H_{n-j, 2 j+d}\right)\right)^{-1} \int_{B} R^{2} .
\end{array}
$$

If we insert the values found above for $c_{d}$, $\operatorname{dim} H_{n, d}$, etc. and simplify we may obtain

$\phi(n, j, n, d) \phi_{*}(n, j, n, d)=\frac{\left[(d-1) ! 2^{j} \Gamma(j+d / 2) / \Gamma(d / 2)(2 n+d-1)\right]^{2} n !(n-j) !}{(n+d-2) !(n+j+d-2) !}$.

In particular the maps $f \rightarrow F f(b, n)$ and $f \rightarrow F_{*} f(b, n)$ are nonsingular from $H_{n, d}$ to $P_{n}(B)$. We will give explicit values for $\phi(n, j, n, d)$ and $\phi_{*}(n, j, n, d)$ in (6) and (7) respectively.

(5) We claim that if $m \neq n$ then $\phi(n, j, m, d)=0$; and if $j \leqslant m$ when $m \neq$ $n$ then $\phi_{*}(n, j, m, d)=0$. (We will leave unresolved the case of $n>j>m$ for $\phi_{*}$.)

Our claim about $\phi$ is equivalent to the following: If $f \in H_{n, d}$ and $m \neq n$ then $F f(b, m)=0$. To see this observe that for each fixed $s$ the function $b \rightarrow$ $e(b, m)(s)$ is in $P_{m}(B)$. Thus $F f(b, m)$ is in $P_{m}(B)$ no matter what $f$ is, $F f(b, m)$ is simply a combination of the functions $e(b, m)(s)$ as $s$ ranges through $S$. Thus if $F f(b, m) \neq 0$ there is a function $g \neq 0$ in $H_{m, d}$ such that $F f(b, m)=F g(b, m)$ (by the nonsingularity of $F$ from $H_{m, d}$ to $P_{m}(B)$ shown in (4)). Now

$$
\begin{aligned}
f_{m}(s) & =\operatorname{dim}\left(H_{m, d}\right) \int_{B} F f(b) e_{*}(b, m)(s) d b \\
& =\operatorname{dim}\left(H_{m, d}\right) \int_{B} F g(b) e_{*}(b, m)(s) d b=g_{m}(s) \not \equiv 0 .
\end{aligned}
$$

But if $f \in H_{n, d}$ and $n \neq m$, this is a contradiction. Our claim about $\phi_{*}$ may be 
rephrased: if $f \in H_{n, d}$ and $m \neq n$ then $F_{*} f(b, m)$ is orthogonal (as a function of $b$ ) to $P_{n}(B)$.

For the proof express an arbitrary element of $P_{m}(B)$ as $F g(b, m)$ where $g \in H_{m, d}$. (This is possible by (4).) Then by Theorem 3.8

$$
0=\int_{S} f g=\operatorname{dim}\left(H_{m, d}\right) \int_{B} F g(b, m) F_{*} f(b, m) d b
$$

which is what we claimed.

(6) Choose $b_{1} \in B$ and let $R(b)=P_{j, d-1}\left(b \cdot b_{1}\right)$. We will compute $F f_{R}\left(b_{1}, n\right)$. This will give us

$$
\phi(n, j, n, d)=\phi(n, j, n, d) R\left(b_{1}\right)=F f_{R}\left(b_{1}, n\right) .
$$

Recall that

$$
f_{R}(\cos \phi a+\sin \phi b)=(\sin \phi)^{i P_{n-j, 2 j+d}}(\cos \phi) P_{j, d-1}\left(b \cdot b_{1}\right) .
$$

If we let $\psi$ be defined on $D$ by

$$
\psi(x+i y)=\left(1-x^{2}\right)^{j / 2} P_{n-j, 2 j+d}(x) P_{j, d-1}\left(y\left(1-x^{2}\right)^{-1 / 2}\right)
$$

then $f_{R}=\psi \circ e(b, 1)$. Thus by Lemmas 5.2 and 5.12

$$
\int_{S} f_{R} e(b, n)=\int_{S} \psi(e(b, 1)) e(b, 1)^{n}=\frac{d-1}{2 \pi} \int \psi(z) z^{n}\left(1-|z|^{2}\right)^{(d-3) / 2} d \lambda(z)
$$

where $\lambda$ denotes Lebesgue measure.

To evaluate this integral, first we change variables from $z \in \mathbf{D}$ to $(\xi, \eta)$ in $[-1,1] \times[-1,1]$ by $z=\xi+i \eta\left(1-\xi^{2}\right)^{1 / 2}$. Then

$$
\begin{gathered}
1-|z|^{2}=\left(1-\xi^{2}\right)\left(1-\eta^{2}\right), \quad d \lambda(z)=\left(1-\xi^{2}\right)^{3 / 2} d \xi d \eta, \\
\psi(z)=\left(1-\xi^{2}\right)^{j / 2} P_{n-j, 2 j+d}(\xi) P_{j, d-1}(\eta)
\end{gathered}
$$

and our integral may be expressed as

$$
\frac{d-1}{2 \pi} \int_{-1}^{1} P_{n-j, d+2 j}(\xi)\left(1-\xi^{2}\right)^{(j+d-2) / 2} g(\xi) d \xi
$$

where

$$
g(\xi)=\int_{-1}^{1}\left(\xi+i \eta\left(1-\xi^{2}\right)^{1 / 2}\right)^{n} P_{j, d-1}(\eta)\left(1-\eta^{2}\right)^{(d-3) / 2} d \eta
$$

We are lead to consider integrals of the form

$$
\int_{-1}^{1}(\alpha+\beta t)^{m} P_{n, d}(t)\left(1-t^{2}\right)^{(d-2) / 2} d t .
$$

Recall that $P_{n, d}(t)=Q_{n, d}(t) / Q_{n, d}(1)$ where 


$$
\begin{aligned}
& Q_{n, d}(t)=\left(1-t^{2}\right)^{-(d-2) / 2}(d / d t)^{n}\left(1-t^{2}\right)^{n+(d-2) / 2}, \\
& Q_{n, d}(1)=(-2)^{n} \Gamma(n+d / 2) / \Gamma(d / 2) .
\end{aligned}
$$

Integration-by-parts gives

$$
\begin{aligned}
& \int_{-1}^{1}(\alpha+\beta t)^{m} P_{n, d}(t)\left(1-t^{2}\right)^{(d-2) / 2} d t \\
&=(-1)^{n}\left(Q_{n, d}(1)\right)^{-1}\left(\frac{m !}{(m-n) !}\right) \beta^{n} \\
& \cdot \int_{-1}^{1}(\alpha+\beta t)^{m-n}\left(1-t^{2}\right)^{n+(d-2) / 2} d t \\
&= C(m, n, d) \beta^{n} I(m, n, d ; \alpha, \beta)
\end{aligned}
$$

where $C(m, n, d)$ denotes the constant, i.e.,

$$
C(m, n, d)=m ! \Gamma(d / 2) / 2^{n} \Gamma(n+d / 2)(m-n) !
$$

and $I$ denotes the integral.

Notice that $I(m, n, d ; \alpha, \beta)$ is a homogeneous polynomial in $(\alpha, \beta)$. Also only even powers of $t$ from the expansion of $(\alpha+\beta t)^{m-n}$ contribute to the integral; consequently $I(m, n, d ; \alpha, \beta)$ contains only even powers of $\beta$.

Now we have immediately that

$$
g(\xi)=C(n, j, d-1)\left(i\left(1-\xi^{2}\right)^{1 / 2}\right)^{j} I\left(n, j, d-1 ; \xi, i\left(1-\xi^{2}\right)^{1 / 2}\right) .
$$

Since $I(n, j, d-1 ; \alpha, \beta)$ is a combination of terms in $\beta^{2 k} \alpha^{n-j-2 k}$ we find that if we substitute $\alpha=\xi$ and $\beta=i\left(1-\xi^{2}\right)^{1 / 2}$ then $I\left(n, j, d-1 ; \xi, i\left(1-\xi^{2}\right)^{1 / 2}\right)$ is the same combination of terms in $\left(\xi^{2}-1\right)^{k} \xi^{n-j-2 i}$. Thus

$$
I\left(n, j, d-1 ; \xi, i\left(1-\xi^{2}\right)^{1 / 2}\right)
$$

is a polynomial $h(\xi)$ in $\xi$ whose highest term is $I(n, j, d-1 ; 1,1) \xi^{n-j}$, and $g(\xi)$ is $\left(1-\xi^{2}\right)^{j / 2} h(\xi)$ multiplied by the constant $C(n, j, d-1)(i)^{j}$.

Now our original integral equals this constant times

$$
\frac{d-1}{2 \pi} \int_{-1}^{1} P_{n-j, d+2 j}(\xi) h(\xi)\left(1-\xi^{2}\right)^{(2 j+d-2) / 2} d \xi .
$$

This may again be evaluated by expressing

$$
P_{n-j, d+2 j}=Q_{n-j, d+2 j} / Q_{n-j, d+2 j}(1)
$$

and integrating-by-parts. The integrand is then a constant multiple of $h^{(n-j)}(\xi)\left(1-\xi^{2}\right)^{n+(d-2) / 2}$. From our considerations above concerning $h$ we see that we would obtain the same result if we simply replaced $h$ by its highest order 
term $I(n, j, d-1 ; 1,1) \xi^{n-j}$. In other words our original integral equals

$$
\begin{gathered}
\frac{d-1}{2 \pi} \int_{-1}^{1} P_{n-j, d+2 j}(\xi) C(n, j, d-1)(i)^{j} I(n, j, d-1 ; 1,1) \\
\cdot \xi^{n-j}\left(1-\xi^{2}\right)^{(2 j+d-2) / 2} d \xi \\
=\frac{d-1}{2 \pi} C(n, j, d-1)(i)^{j} I(n, j, d-1 ; 1,1) \\
\cdot C(n-j, n-j, d+2 j) I(n-j, n-j, d+2 j ; 0,1) . \\
I(n, j, d-1 ; 1,1)=\int_{-1}^{1}(1+t)^{n-j}\left(1-t^{2}\right)^{j+(d-3) / 2} d \xi \\
=\int_{-1}^{1}(1+t)^{n+(d-3) / 2}(1-t)^{j+(d-3) / 2} d t \\
=2^{n+j+d-2} \Gamma(n+(d-1) / 2) \Gamma(j+(d-1) / 2) \Gamma(n+j+d-1) . \\
I(n-j, n-j, d+2 j ; 0,1)=\int_{-1}^{1}\left(1-t^{2}\right)^{n+(d-2) / 2} d t \\
=2^{2 n+d-1} \Gamma(n+d / 2)^{2} / \Gamma(2 n+d) .
\end{gathered}
$$

If we combine the various formulae for $C(n, j, d-1)$, etc., and cancel some factors we get

$$
\frac{(i)^{j} 2^{2 n+j+2 d-3} n ! \Gamma((d+1) / 2) \Gamma(j+d / 2) \Gamma(n+(d-1) / 2) \Gamma(n+d / 2)}{\pi \Gamma(n+j+d-1) \Gamma(2 n+d)}
$$

for $\phi(n, j, n, d)$. If we use the duplication formula for $\Gamma$ the factor $\Gamma(n+(d-1) / 2) \Gamma(n+d / 2) / \Gamma(2 n+d)$ becomes $\pi^{1 / 2} / 2^{2 n+d-2}(2 n+d-1)$. Thus finally

$$
\phi(n, j, n, d)=\frac{(i)^{j} 2^{j+d-1} n ! \Gamma((d+1) / 2) \Gamma(j+d / 2)}{\pi^{1 / 2}(n+j+d-2) !(2 n+d-1)} .
$$

(7) If we combine this with the result for $\phi \phi_{*}$ found in (4) we obtain

$$
\begin{aligned}
\phi_{*}(n, j, n, d) & =\frac{(-i)^{j} 2^{j+d-1} \Gamma(j+d / 2) \Gamma((d+1) / 2)(n-j)}{\pi^{1 / 2}(n+d-2)(2 n+d-1)} \\
& =\frac{(-1)^{j}(n-j) !(n+j+d-2) !}{n !(n+d-2) !} \phi(n, j, n, d) .
\end{aligned}
$$

This is most easily seen by rewriting $\phi \phi_{*}$ using

$$
(d-1) ! / \Gamma(d / 2)=\Gamma((d+1) / 2) 2^{d-1} / \pi^{1 / 2}
$$


to obtain

$$
\begin{aligned}
& \phi(n, j, n, d) \phi_{*}(n, j, n, d) \\
& \quad=\frac{\left[2^{j+d-1} \Gamma((d+1) / 2) \Gamma(j+d / 2) / \pi^{1 / 2}(2 n+d-1)\right]^{2} n !(n-j) !}{(n+d-2) !(n+j+d-2) !} .
\end{aligned}
$$

(8) In a different direction fix $b_{1} \in B$ and let $f=e\left(b_{1}, n\right)$. We propose to calculate $F_{*} f(b, n)$. In principle this is possible using (7). However, we propose a different approach.

Let $h_{n}\left(b_{1}, b\right)=F_{*} e\left(b_{1}, n\right)(b, n)$. By $K$-equivariance of $F_{*}, h_{n}\left(k b_{1}, k b\right)=$ $h_{n}\left(b_{1}, b\right)$ so there is a function $\delta_{n}$ on $[-1,1]$ such that $h_{n}\left(b_{1}, b\right)=\delta_{n}\left(b_{1} \cdot b\right)$.

Now by (3) $h_{n}\left(b_{1}, b\right)$ is a polynomial in $b$ of degree less or equal to $n$. Then $\delta_{n}$ is a polynomial of the same degree on $[-1,1]$.

By the inversion formula for $F_{*}$

$$
\begin{aligned}
e\left(b_{1}, n\right)(s) & =\operatorname{dim}\left(H_{n, d}\right) \int_{B} F_{*} e\left(b_{1}, n\right)(b, n) e(b, n)(s) d b \\
& =\operatorname{dim}\left(H_{n, d}\right) \int_{B} \delta_{n}\left(b_{1} \cdot b\right) e(b, n)(s) d b .
\end{aligned}
$$

The fact that $F$ maps $H_{n, d}$ onto $P_{n}(B)$ (see (3) above) implies that as $s$ ranges through $S, e(b, n)(s)$ (as a function of $b \in B$ ) ranges through a spanning set in $P_{n}(B)$. Thus for $Q \in P_{n}(B)$ we have

$$
Q\left(b_{1}\right)=\operatorname{dim}\left(H_{n, d}\right) \int_{B} \delta_{n}\left(b_{1} \cdot b\right) Q(b) d b .
$$

Consequently $\operatorname{dim}\left(H_{n, d}\right) \delta_{n}\left(b_{1} \cdot b\right)$ is the "Dirichlet kernel" of degree $n$ for harmonic analysis on $B$. It may be given explicitly as $\Sigma_{j=0}^{n}\left(\operatorname{dim} H_{j, d-1}\right) P_{j, d-1}\left(b_{1} \cdot b\right)$.

To summarize, $F_{*} e\left(b_{1}, n\right)(b, n)=\delta_{n}\left(b_{1} \cdot b\right)$ where

$$
\delta_{n}(t)=\left(\operatorname{dim} H_{n, d}\right)^{-1} \sum_{j=0}^{n}\left(\operatorname{dim} H_{j, d-1}\right) P_{j, d-1}(t) .
$$

By the Christoffel-Darboux formula [1, p. 159] we then have

$$
\delta_{n}(t)=L_{n, d}\left(P_{n+1, d-1}(t)-P_{n, d-1}(t)\right) /(t-1)
$$

where $L_{n, d}$ is a constant so chosen that $\delta_{n}(1)=1$. (This choice of $L_{n, d}$ follows from $\operatorname{dim} H_{n, d}=\Sigma_{j=0}^{n} \operatorname{dim} H_{j, d-1}$.)

$$
\begin{aligned}
L_{n, d}^{-1} & =\lim _{t \rightarrow 1}\left(P_{n+1, d-1}(t)-P_{n, d-1}(t)\right) /(t-1) \\
& =P_{n+1, d-1}^{\prime}(1)-P_{n, d-1}^{\prime}(1) .
\end{aligned}
$$

The functions $y(t)=P_{m, d-1}(t)$ satisfy 


$$
\left(1-t^{2}\right) y^{\prime \prime}-(d-1) t y^{\prime}+m(m+d-2) y=0 \text {. }
$$

Consequently $P_{m, d-1}^{\prime}(1)=m(m+d-2) /(d-1)$, and $L_{n, d}=(d-1) /(2 n+d-1)$.

\section{REFERENCES}

1. A. Erdelyi et al., Higher transcendental functions. Vol. 2, McGraw-Hill, New York, 1953. MR 15, 419.

2. S. Helgason, $A$ duality for symmetric spaces with applications to group representations, Advances in Math. 5 (1970), 1-154. MR 41 \#8587.

3. C. E. F. Müller, Spherical harmonics, Lecture Notes in Math., no. 17, SpringerVerlag, Berlin and New York, 1966. MR 33 \#7593.

4. E. T. Whittaker and G. N. Watson, $A$ course of modern analysis, 4th ed., Cambridge Univ. Press, Cambridge, 1927; reprint, 1962. MR 31 \#2375.

DEPARTMENT OF MATHEMATICS, NORTHEASTERN UNIVERSITY, BOSTON, MASSACHUSETTS 02115 